\title{
SYSTEMS OF DIAGRAM CATEGORIES AND K-THEORY. II
}

\author{
GRIGORY GARKUSHA
}

\begin{abstract}
The additivity theorem for dérivateurs associated to complicial biWaldhausen categories is proved. Also, to any exact category in the sense of Quillen a $K$-theory space is associated. This $K$-theory is shown to satisfy the additivity, approximation and resolution theorems.
\end{abstract}

\section{INTRODUCTION}

It is well known due most recently to work of Schlichting [18] that, in general, there is no $K$-theory for triangulated categories satisfying localization and reconstructing Quillen's $K$-theory of an exact category from its derived category. There are two approaches to replace the naive notion of derived category by something richer, from which the $K$-theory might be obtained by some explicit construction. One approach, suggested by Dwyer and Kan [3, 4], is called the "simplicial localization". Toën and Vezzosi [23] define a $K$ theory functor on the level of $S$-categories and prove that, when applied to the simplicial localization of an appropriate Waldhausen category $\mathscr{C}$, this construction yields a spectrum which is weakly equivalent to the Waldhausen's $K$-theory spectrum of $\mathscr{C}$. The other, following definitions of Grothendieck [7], Heller [8] and Franke [5] is called the "system of diagram categories" or the "dérivateur" (in French): given a closed model category $\mathscr{C}$, one takes a large system of index categories $I$, and forms the system of derived categories of the diagram categories $\mathscr{C}^{I}$. One can introduce an analogue of the Q.-construction (see [14]) or Waldhausen's $S$.-construction [6] for them which might form a suitable definition for the $K$-theory of a system of diagram categories or a dérivateur. Both definitions give equivalent $K$-theories by [2].

Maltsiniotis [14] opens three conjectures, the third of which says that the $K$-theory of a dérivateur (or a system of diagram categories) satisfies the additivity theorem. A weaker version of additivity has been shown in [6]. One could try to adapt Waldhausen's [25] or McCarthy's [15] proof of additivity in this context to show the strong form of additivity. At the first glance, the machinery of dérivateurs seems to have some of the necessary ingredients. However one thing always goes wrong when constructing a homotopy; one lacks a component which seems to be not deducible from dérivateurs in general (see details at the end of section (4).

In this paper we prove the strong form of additivity for dérivateurs associated to complicial biWaldhausen categories in the sense of Thomason [22] (see the precise definitions in section 47. Thus we can find the lacking ingredient in this case. Experts will probably be able to show additivity for the dérivateurs represented by closed model categories.

2000 Mathematics Subject Classification. Primary 19D99.

Key words and phrases. Systems of diagram categories, Dérivateurs of Grothendieck, K-theory.

Supported by the ICTP Research Fellowship. 
Such dérivateurs seem to contain all the necessary information for this. If so, it will be justifiable to say that the third Maltsiniotis conjecture is true for the dérivatuers "having a model". In fact, all interesting dérivatuers we have in nature arise in this fashion.

To any exact category $\mathscr{E}$ one associates the dérivateur

$$
\mathbf{D}^{b}(\mathscr{E}): I \longmapsto D^{b}(\mathscr{E} I)
$$

sending an index category $I$ to the derived category of the exact diagram category $\mathscr{E} I$. It is very interesting to study its $K$-theory space $K\left(\mathbf{D}^{b}(\mathscr{E})\right)$. This $K$-theory is shown to satisfy the additivity, approximation and resolution theorems.

Organization of the paper. After fixing some notation and terminology (in 2.1] and 2.2), we formulate several lemmas (in 2.3) which are of great utility in proving the additivity theorem in section 3, Our main result is then formulated in section 4, that dealing with additivity for dérivateurs associated to complicial biWaldhausen categories (Theorem 4.5). Then comes section 5 in which the $K$-theory space $K\left(\mathbf{D}^{b}(\mathscr{E})\right)$ is studied. We also prove there a couple of results which are of independent interest. The necessary facts about dérivateurs and their $K$-theory are given in Addendum.

Acknowledgement. I would like to thank Professor Haynes Miller and an anonymous referee for helpful suggestions concerning the material of the paper.

\section{COCHAIN COMPLEXES, HOMOTOPIES, DERIVED CATEGORIES}

In this section we collect some necessary facts about cochain complexes and derived categories.

2.1. Definition of the derived category. An exact category is an additive category $\mathscr{A}$ with a collection of exact sequences $\{E \longmapsto F \rightarrow G\}$ where the first morphism $E \longmapsto F$ appearing in those exact sequences are called admissible monomorphisms and the second ones admissible epimorphisms. They have to satisfy a couple of natural axioms (e.g. see [22]). Let $\mathscr{C}=C^{b}(\mathscr{A})$ denote the category of bounded complexes in an exact category $\mathscr{A}$. Recall that $\mathscr{A}$ can be embedded as a full subcategory in an abelian category $\mathscr{B}$ in such a way that a sequence in $\mathscr{A}$ is exact if and only if it is exact in $\mathscr{B}$. If $\mathscr{A}$ is idempotent complete (or even less) this embedding can be chosen in a way that any map in $\mathscr{A}$ which becomes an epimorphism in $\mathscr{B}$ was already an admissible epimorphism in $\mathscr{A}$ (see [22]).

Let $\mathscr{A}$ be an exact category. Its bounded derived category $D^{b}(\mathscr{A})$ is constructed as follows (we follow here Keller's definition [12]).

Let $H^{b}(\mathscr{A})$ be the homotopy category of the category of bounded complexes $\mathscr{C}=$ $C^{b}(\mathscr{A})$, i.e., the quotient category of $\mathscr{C}$ modulo homotopy equivalence. Let $A c(\mathscr{A})$ denote the full subcategory of $H^{b}(\mathscr{A})$ consisting of acyclic complexes. A complex

$$
X^{n} \longrightarrow X^{n+1} \longrightarrow X^{n+2}
$$

is called acyclic if each map $X^{n} \longrightarrow X^{n+1}$ decomposes in $\mathscr{A}$ as $X^{n} \stackrel{e_{n}}{\rightarrow} D^{n} \stackrel{m_{n}}{\longrightarrow} X^{n+1}$ where $e_{n}$ is an epimorphism and $m_{n}$ is a monomorphism in such a way that $D^{n} \longmapsto$ $X^{n+1} \stackrel{e_{n+1}}{\rightarrow} D^{n+1}$ is an exact sequence. 
If an exact category is idempotent complete then every contractible complex is acyclic. Denote by $\mathscr{N}=\mathscr{N}(\mathscr{A})$ the full subcategory of $H^{b}(\mathscr{A})$ whose objects are the complexes isomorphic in $H^{b}(\mathscr{A})$ to acyclic complexes. There is another description of $\mathscr{N}$. Let $\mathscr{A} \longrightarrow \tilde{A}$ be the universal additive functor to an idempotent complete exact category $\tilde{\mathscr{A}}$. It is exact and reflects exact sequences, and $\mathscr{A}$ is closed under extensions in $\tilde{\mathscr{A}}$ (see [22, A.9.1]). The class of acyclic complexes in $\tilde{\mathscr{A}}$ is closed under homotopy equivalence. It follows that a complex with entries in $\mathscr{A}$ belongs to $\mathscr{N}$ if and only if its image in $H^{b}(\tilde{\mathscr{A}})$ is acyclic. The category $\mathscr{N}(\tilde{\mathscr{A}})=A c(\tilde{\mathscr{A}})$ is a thick subcategory in $H^{b}(\tilde{\mathscr{A}})$. Note that a complex over $\tilde{\mathscr{A}}$ is acyclic if and only if it has trivial homology computed in an appropriate ambient abelian category $\mathscr{B}$ (see above). It follows that $\mathscr{N}$ is a thick subcategory in $H^{b}(\mathscr{A})$. Denote by $\Sigma$ the multiplicative system associated to $\mathscr{N}$ and call the elements of $\Sigma$ quasi-isomorphisms. A map $s$ is a quasi-isomorphism if and only if in any triangle

$$
L \stackrel{s}{\longrightarrow} M \longrightarrow N \longrightarrow L[1]
$$

the complex $N$ belongs to $\mathscr{N}$.

The derived category is defined as

$$
D^{b}(\mathscr{A})=H^{b}(\mathscr{A}) / \mathscr{N}=H^{b}(\mathscr{A})\left[\Sigma^{-1}\right] .
$$

Clearly, a map is a quasi-isomorphism if and only if its image in $C^{b}(\tilde{\mathscr{A}})$ is a quasiisomorphism and if and only if its image in $D^{b}(\mathscr{A})$ is an isomorphism.

We shall work a lot with derived categories of diagram exact categories $\mathscr{A}^{I}$ where $I$ is a small category. It is easily seen that a cochain map $f: A \longrightarrow B$ in $C^{b}\left(\mathscr{A}^{I}\right)$ is a quasi-isomorphisms if and only if each $f_{i}: A_{i} \longrightarrow B_{i}, i \in I$, is so in $C^{b}(\mathscr{A})$.

2.2. Homotopy pullbacks and homotopy pushouts. Let $f: F \longrightarrow A$ and $g: G \longrightarrow A$ be cochain maps. One has a canonical homotopy pullback

$$
\begin{gathered}
\left(F \prod_{A}^{h} G\right)^{n}=F^{n} \oplus A^{n-1} \oplus G^{n} \\
d(x, a, y)=\left(d_{F} x,-d_{A} a+f x-g y, d_{G} y\right) .
\end{gathered}
$$

(We describe $d$ as if objects of $\mathscr{A}$ had "elements", by the standard abuse). The square

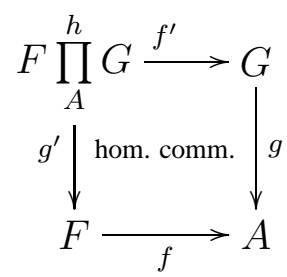

with $f^{\prime}(x, a, y)=y$ and $g^{\prime}(x, a, y)=x$ is homotopy commutative, that is $g f^{\prime} \sim f g^{\prime}$. Note that $f^{\prime}$ is a quasi-isomorphism whenever $f$ is. Cochain maps from a complex $C$ to this canonically homotopy pullback correspond bijectively to data $(h, p, k)$ where $h: C \longrightarrow$ $F$ and $p: C \longrightarrow G$ are cochain maps and $k$ is a cochain homotopy $f h \sim g p: C \longrightarrow A$. Thus $k$ consists of maps $C^{n} \longrightarrow A^{n-1}$ for all $n$ such that $d k+k d=f h-g p$. To $(h, p, k)$ corresponds the cochain map $t: C \longrightarrow F \prod_{A}^{h} G$ defined as $t(c)=(h c, k c, p c)$. Then 
$f^{\prime} t=p$ and $g^{\prime} t=h$. When $f: F \longrightarrow A$ is the identity map, the canonically homotopy pullback is the mapping cocylinder $\operatorname{Cocyl}(g)$ of $g: G \longrightarrow A$.

Dually, given $f: A \longrightarrow F$ and $g: A \longrightarrow G$ the canonically homotopy pushout is the complex defined by

$$
\begin{gathered}
\left(F \coprod_{A}^{h} G\right)^{n}=F^{n} \oplus A^{n+1} \oplus G^{n} \\
d(x, a, y)=\left(d_{F} x+f a,-d_{A} a, d_{G} y-g a\right) .
\end{gathered}
$$

This indeed has all the dual properties as the homotopy pullback. As special cases, when $f: A \longrightarrow F$ is the identity map, the homotopy pushout is the mapping cylinder $\operatorname{Cyl}(g)$ of $g: A \longrightarrow G$. If $f: A \longrightarrow F=0$ is the map to 0 , the homotopy pushout is the mapping cone $C(g)$ of $g: A \longrightarrow G$.

Let $w \mathscr{C}$ denote the category whose objects are those of $\mathscr{C}$ and morphisms are quasiisomorphisms. It is a complical biWaldhausen category (see definitions in [22, 25]). It has also cylinder and cocylinder functors satisfying the cylinder and cocylinder axioms.

2.3. Getting rid of homotopy commutative squares. Results of this technical paragraph are of great utility in proving the "additivity theorem" in the next section. To construct a homotopy in that proof we will want to replace some homotopy commutative diagrams by strictly commutative ones. Given a non-negative integer $n$, by $\Delta^{n}$ denote the totally ordered set $\{0<1<\cdots<n\}$.

Suppose we are given a homotopy commutative square with entries $\left(X_{0}, Y, A_{0}, A_{1}\right)$

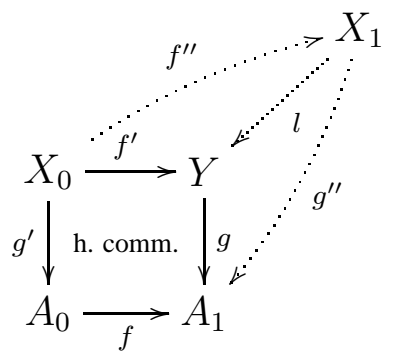

We want to replace it by a strictly commutative square with entries $\left(X_{0}, X_{1}, A_{0}, A_{1}\right)$.

Let $X_{1}=\operatorname{Cocyl}(g)$; then $g l \sim g^{\prime \prime}$. Note that a homotopy is given by the maps $z^{n}$ : $X_{1}^{n} \longrightarrow A_{1}^{n-1}$ mapping $(x, a, y) \in X_{1}^{n}$ to $a$. Since $g f^{\prime} \sim f g^{\prime}$ there is a map $f^{\prime \prime}$ : $X_{0} \longrightarrow X_{1}$ such that $l f^{\prime \prime}=f^{\prime}$ and $g^{\prime \prime} f^{\prime \prime}=f g^{\prime}$. A cochain map $f^{\prime \prime}$ is defined by $f^{\prime \prime}(x)=$ $\left(f^{\prime}(x), k(x), f g^{\prime}(x)\right)$ where maps $k^{n}: X_{0}^{n} \longrightarrow A_{1}^{n-1}$ give a homotopy $g f^{\prime} \sim f g^{\prime}$.

Lemma 2.1. Suppose that in the diagram

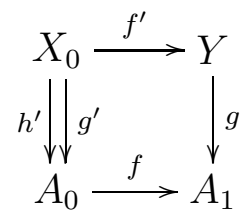


the maps $h^{\prime}, g^{\prime}$ are homotopic and the square with $g^{\prime}$ deleted is genuinely commutative. Then one can produce a pair of genuinely commutative squares

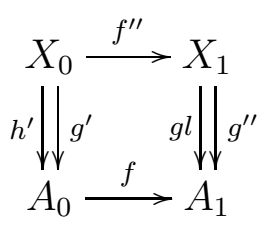

such that the map $\left(g^{\prime}, g^{\prime \prime}\right): X=\left(X_{0} \stackrel{f^{\prime \prime}}{\longrightarrow} X_{1}\right) \longrightarrow A=\left(A_{0} \stackrel{f}{\longrightarrow} A_{1}\right)$ is homotopic to the map $\left(h^{\prime}, g l\right): X \longrightarrow A$ in $\mathscr{C}^{\Delta^{1}}=C^{b}\left(\mathscr{A}^{\Delta^{1}}\right)$.

Proof. Since $f g^{\prime} \sim g f^{\prime}$ one can construct a diagram as above with $X_{1}=\operatorname{Cocyl}(g)$. By construction, $f^{\prime \prime}(x)=\left(f^{\prime}(x), f m(x), f g^{\prime}(x)\right)$ where maps $m^{n}: X_{0}^{n} \longrightarrow A_{0}^{n-1}$ yield a homotopy $g^{\prime} \sim h^{\prime}$. For any $n$ the square

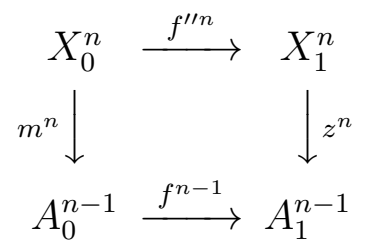

is commutative and the maps $\left(m^{n}, z^{n}\right): X^{n} \longrightarrow A^{n-1}$ give the desired homotopy.

A map $X \longrightarrow Y$ in $D^{b}(\mathscr{A})$ is the equivalence class of a diagram in $C^{b}(\mathscr{A})$

$$
X \stackrel{s}{\longleftarrow} Z \stackrel{f}{\longrightarrow} Y
$$

with $s$ a quasi-isomorphism. It is equivalent to $X \stackrel{t}{\longleftarrow} W \stackrel{g}{\longrightarrow} Y$ if these fit into a homotopy commutative diagram

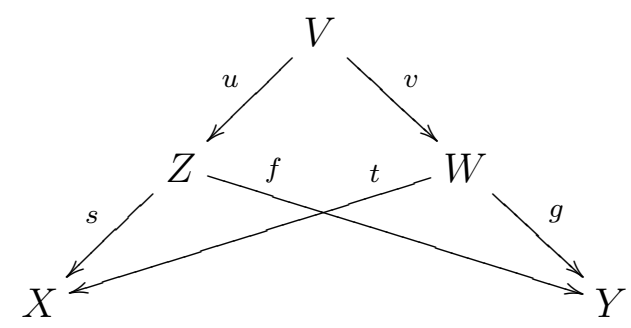

with $u$ and $v$ quasi-isomorphisms.

Lemma 2.2. Let $f s^{-1}: A \longrightarrow C$ be a map in $D^{b}\left(\mathscr{A}^{\Delta^{1}}\right)$ represented by a commutative diagram

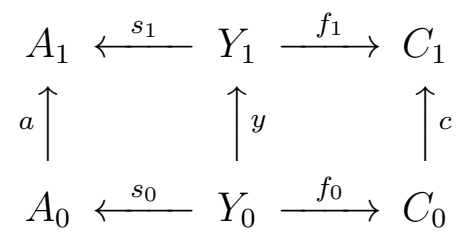


with $s_{0}, s_{1}$ quasi-isomorphisms and let $A_{0} \stackrel{t}{\longleftarrow} U \stackrel{h}{\longrightarrow} C_{0}$ be another representative for $A_{0} \stackrel{f_{0} s_{0}^{-1}}{\longrightarrow} C_{0}$ in $D^{b}(\mathscr{A})$ with a common denominator

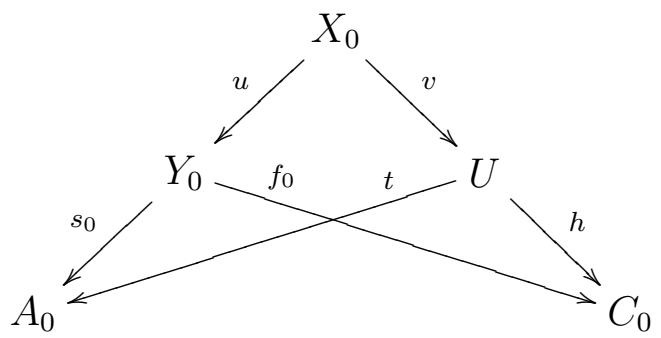

Then there exists a complex $X_{1}$ and a commutative diagram

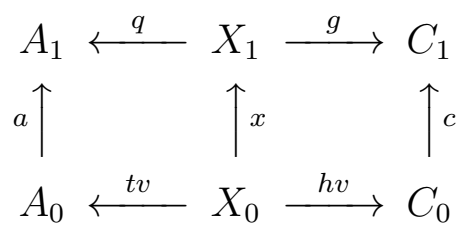

representing the same morphism $f s^{-1}$ in $D^{b}\left(\mathscr{A}^{\Delta^{1}}\right)$. If $f_{0}, f_{1}$ are quasi-isomorphisms then so is $g$. Moreover, $X_{1}$ can be chosen in such a way that $x$ is a monomorphism in $\mathscr{C}$.

Proof. Applying the preceding lemma first to the diagram

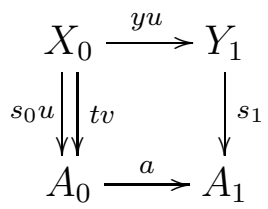

one obtains a diagram

$$
\begin{gathered}
X_{0} \stackrel{p_{1}}{\longrightarrow} Y_{2} \\
s_{0} u\left\|_{t v} \quad s_{1} l_{1}\right\|_{\Downarrow} q_{1} \\
A_{0} \stackrel{a}{\longrightarrow} A_{1}
\end{gathered}
$$

and then to the diagram

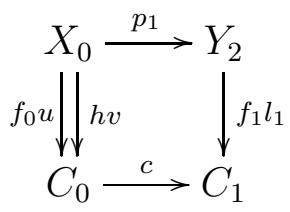

resulting a diagram

$$
\begin{aligned}
& X_{0} \stackrel{x}{\longrightarrow} X_{1} \\
& f_{0} u \Downarrow h v \quad f_{1} l_{1} l_{2} \Downarrow \downarrow g \\
& C_{0} \stackrel{c}{\longrightarrow} C_{1}
\end{aligned}
$$

We put $q=q_{1} l_{2}$. The diagram (2) is constructed. It is equivalent to (1), hence represents the map $f s^{-1}$ in $D^{b}\left(\mathscr{A}^{\Delta^{1}}\right)$. The fact that the map $g$ is a quasi-isomorphism if $f_{0}, f_{1}$ are is obvious. 
Finally, to show that $X_{1}$ can be chosen in such a way that $x$ is a monomorphism in $\mathscr{C}$ it is enough to observe that any cochain map $z: X_{0} \longrightarrow Y_{3}$ is the composite $X_{0} \stackrel{x}{\longrightarrow} X_{1} \stackrel{w}{\longrightarrow}$ $Y_{3}$ of a monomorphism $x$ followed by a quasi-isomorphism $w$ and $X_{1}=\operatorname{Cyl}(z)$.

Let $\square$ be the poset $\Delta^{1} \times \Delta^{1}$ and let $\ulcorner\subset \square$ be the subposet $\square \backslash(1,1)$. Then the exact diagram category $\mathscr{A}\ulcorner$ consists of the diagrams in $\mathscr{A}$

$$
A_{(1,0)} \longleftarrow A_{(0,0)} \longrightarrow A_{(0,1)}
$$

Let $\widetilde{\mathscr{A}}\left\ulcorner\right.$ be the full subcategory in $\mathscr{A}\left\ulcorner\right.$ with $A_{(0,0)} \longrightarrow A_{(0,1)}$ an admissible monomorphism. In turn, the exact diagram category $\mathscr{A}^{\square}$ consists of the commutative squares

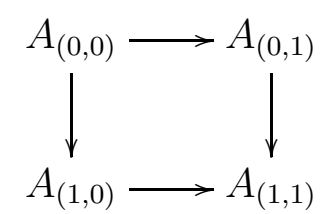

Denote by $\widetilde{A}^{\square}$ the full subcategory in $\mathscr{A}^{\square}$ with $A_{(0,0)} \longrightarrow A_{(0,1)}$ and $A_{(1,0)} \longrightarrow A_{(1,1)}$ admissible monomorphisms in $\mathscr{A}$ and the square above is cocartesian.

It follows that $\widetilde{\mathscr{A}}\ulcorner$ is an exact subcategory of $\mathscr{A}\ulcorner$ and $\widetilde{\mathscr{A}}$ is an exact subcategory of $\mathscr{A}^{\square}$. Therefore one can consider their derived categories $D^{b}\left(\widetilde{\mathscr{A}^{\square}}\right)$ and $D^{b}(\widetilde{\mathscr{A}}\ulcorner)$. We claim that they are naturally equivalent. To see this, consider the functor $i_{\ulcorner}^{*}: \widetilde{\mathscr{A}^{\square} \longrightarrow \widetilde{\mathscr{A}}\ulcorner}$ taking a square

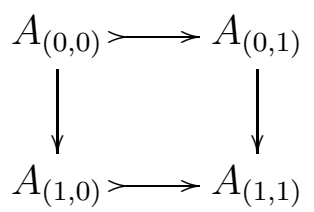

to $A_{(1,0)} \leftarrow A_{(0,0)} \longmapsto A_{(0,1)}$ as well as the functor $j: \widetilde{\mathscr{A}}\ulcorner\longrightarrow \widetilde{\mathscr{A}}$ taking a diagram $A_{(1,0)} \leftarrow A_{(0,0)} \longmapsto A_{(0,1)}$ to

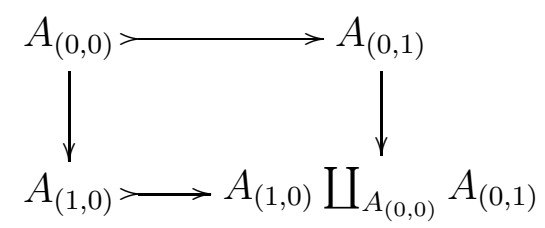

Then $i_{\ulcorner}^{*}$ and $j$ are exact functors and plainly mutual inverses with $i_{\ulcorner}^{*} j=\mathrm{id}$. These induce the desired equivalence of derived categories.

Corollary 2.3. Given two squares of cochain complexes

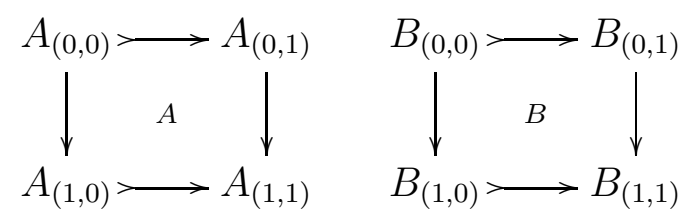

in $D^{b}\left(\widetilde{\mathscr{A}^{\square}}\right)$ and a morphism $\alpha: i_{\ulcorner}^{*}(A) \longrightarrow i_{\ulcorner}^{*}(B)$ in $D^{b}(\widetilde{\mathscr{A}}\ulcorner)$, there exists a unique map $a: A \longrightarrow B$ such that $i_{r}^{*}(a)=\alpha$. If $\alpha$ is an isomorphism then so is $a$. 


\section{THE ADDITIVITY THEOREM}

In this section we prove a sort of the additivity theorem. It assumes the role of a basic result in algebraic $K$-theory. We refer the reader to Staffeldt's work [21]. The author knows two proofs of that theorem for Waldhausen's categories: by Waldhausen [25] and by McCarthy [15]. We shall follow Waldhausen's proof.

Waldhausen [24, 25] constructs a simplicial exact category $S . \mathscr{A}=\left\{S_{n} \mathscr{A}\right\}_{n \geqslant 0}$ in which the face and the degeneracy maps are exact functors. Let $\operatorname{Ar} \Delta^{n}$ be the poset of pairs $(i, j), 0 \leqslant i \leqslant j \leqslant n$, where $(i, j) \leqslant\left(i^{\prime}, j^{\prime}\right)$ if and only if $i \leqslant i^{\prime}$ and $j \leqslant j^{\prime}$. An object of $S_{n} \mathscr{A}$ is a functor $A: \operatorname{Ar} \Delta^{n} \longrightarrow \mathscr{A}$ such that $A_{i i}=0$ and

$$
A_{i j} \longrightarrow A_{i k} \longrightarrow A_{j k}
$$

is a short exact sequence in $\mathscr{A}$ for any $0 \leqslant i \leqslant j \leqslant k \leqslant n$. Observe (exercise!) that a cochain map $f: A \longrightarrow A^{\prime}$ in $C^{b}\left(S_{n} \mathscr{A}\right)$ is a quasi-isomorphism if and only if each $f_{i j}: A_{i j} \longrightarrow A_{i j}^{\prime}$ is so in $C^{b}(\mathscr{A})$.

For any $n \geqslant 1$ the exact category $S_{n} \mathscr{A}$ is equivalent to the exact category $F_{n-1} \mathscr{A}$ of composable monomorphisms in $\mathscr{A}$

$$
A_{0} \longmapsto A_{1} \longmapsto \cdots \longmapsto A_{n-1} .
$$

This equivalence is given by the the exact functor forgetting quotients.

Denote by $i$.S. $\mathscr{A}$ the bisimplicial set

$$
\Delta^{m} \times \Delta^{n} \longmapsto i_{m} \mathbf{S}_{n} \mathscr{A}=i_{m} D^{b}\left(S_{n} \mathscr{A}\right) .
$$

The $(m, n)$-simplices are represented by the strings of isomorphisms in $\mathbf{S}_{n} \mathscr{A}=D^{b}\left(S_{n} \mathscr{A}\right)$

$$
A_{0} \stackrel{\sim}{\longrightarrow} A_{1} \stackrel{\sim}{\longrightarrow} \cdots \stackrel{\sim}{\longrightarrow} A_{m} .
$$

Note that every exact functor $f: \mathscr{A} \longrightarrow \mathscr{A}^{\prime}$ induces a simplicial map $f_{*}: i$. S. $\mathscr{A} \longrightarrow$ $i$.S. $\mathscr{A}^{\prime}$. We also observe that coproduct gives a unitial and associative $H$-space structure to $|i . \mathrm{S} . \mathscr{A}|$ via the map

$$
|i . \mathbf{S} . \mathscr{A}| \times|i . \mathbf{S} . \mathscr{A}| \stackrel{\sim}{\longrightarrow}|i . \mathbf{S} . \mathscr{A} \times i . \mathbf{S} . \mathscr{A}| \stackrel{\amalg}{\longrightarrow}|i . \mathbf{S} . \mathscr{A}| .
$$

The category $\mathbf{S}_{0} \mathscr{A}$ is the trivial category with one object and one morphism. Hence the geometric realization $\left|i . \mathbf{S}_{0} \mathscr{A}\right|$ is the one-point space. The category $\mathbf{S}_{1} \mathscr{A}$ is isomorphic to the derived category $D^{b}(\mathscr{A})$. Hence the category of isomorphisms $i \mathbf{S}_{1} \mathscr{A}$ may be identified to $i D^{b}(\mathscr{A})$.

Consider $\mid i$.S. $\mathscr{A} \mid$. The "1-skeleton" in the $S$-direction is obtained from the "0-skeleton" (which is $\left|i . \mathbf{S}_{0} \mathscr{A}\right|$ ) by attaching of $\left|i . \mathbf{S}_{1} \mathscr{A}\right| \times|\Delta[1]|$ (where $|\Delta[1]|$ denotes the topological space 1-simplex). It follows that the "1-skeleton" is naturally isomorphic to the suspension $S^{1} \wedge\left|i . D^{b}(\mathscr{A})\right|$. One obtains an inclusion $S^{1} \wedge\left|i . D^{b}(\mathscr{A})\right| \longrightarrow|i . \mathbf{S} . \mathscr{A}|$, and by adjointness an inclusion of $\left|i . D^{b}(\mathscr{A})\right|$ into the loop space of $|i . \mathrm{S} . \mathscr{A}|$,

$$
\left|i . D^{b}(\mathscr{A})\right| \longrightarrow \Omega \mid i . \text { S. } \mathscr{A} \mid \text {. }
$$

We can apply the $S$.-construction to produce a bisimplicial category, S.S. $\mathscr{A}=D^{b}(S . S . \mathscr{A})$, and more generally a multisimplicial category, $\mathbf{S} \cdot{ }^{n} \mathscr{A}=D^{b}\left(S{ }^{n} \mathscr{A}\right)$. There results a spectrum

$$
n \longmapsto\left|i . \mathbf{S}^{n} \mathscr{A}\right|
$$

whose structure maps are defined as the map $\left|i . D^{b}(\mathscr{A})\right| \longrightarrow \Omega \mid i$. S. $\mathscr{A} \mid$ above. 
It turns out that the spectrum is a $\Omega$-spectrum beyond the first term (the additivity theorem is needed to show this, below). As the spectrum is connective (the $n$th term is $(n-1)$-connected) an equivalent assertion is that in the sequence

$$
\left|i . D^{b}(\mathscr{A})\right| \longrightarrow \Omega \mid i . \text { S.A }|\longrightarrow \Omega \Omega| i \text {.S.S. } \mathscr{A} \mid \longrightarrow \cdots
$$

all maps except the first are homotopy equivalences.

Let $\mathscr{A}$ be an exact category and let $\mathscr{E}$ be its extension category. There are three natural simplicial maps $s_{*}, t_{*}, q_{*}: i . \mathbf{S} . \mathscr{E} \longrightarrow i . \mathrm{S} . \mathscr{A}$ induced by $s, t, q: \mathscr{E} \longrightarrow \mathscr{A}$ that take a short exact sequence

$$
A \longrightarrow C \longrightarrow B
$$

to $A, C$ and $B$ respectively.

Theorem 3.1 (Additivity). Let $\mathscr{A}$ be an exact category and let $\mathscr{E}$ be its extension category. Then the map

$$
i . \mathbf{S} . \mathscr{E} \stackrel{\left(s_{*}, q_{*}\right)}{\longrightarrow} i . \mathbf{S} . \mathscr{A} \times i . \mathbf{S} . \mathscr{A}
$$

is a homotopy equivalence.

Before proving the theorem we recall the reader certain simplicial facts.

Lemma 3.2 ([20]). Let $X . . \longrightarrow Y$.. be a map of bisimplicial sets. Suppose that for every $n$, the map $X_{\cdot_{n}} \longrightarrow Y_{{ }_{n}}$ is a homotopy equivalence. Then $X . . \longrightarrow Y$.. is a homotopy equivalence.

Lemma $3.3([24])$. Let $X . . \longrightarrow Y$.. $\longrightarrow Z$.. be a sequence of bisimplicial sets so that $X . . \longrightarrow Z$.. is constant. Suppose that $X_{\cdot_{n}} \longrightarrow Y_{\cdot_{n}} \longrightarrow Z_{\cdot_{n}}$ is a fibration up to homotopy, for every $n$. We also require a compatibility with $n$. Suppose further that $Z_{{ }_{n} n}$ is connected for every $n$. Then $X . . \longrightarrow Y$.. $\longrightarrow Z$.. is a fibration up to homotopy.

Let $\Delta[n]$ denote the simplicial set standard $n$-simplex, $\Delta^{m} \longmapsto \operatorname{Hom}_{\Delta}\left(\Delta^{m}, \Delta^{n}\right)$. Let $f: X \longrightarrow Y$ be a map of simplicial sets and let $y$ be a $n$-simplex of $Y$. Define a simplicial set $f /(n, y)$ as the pullback

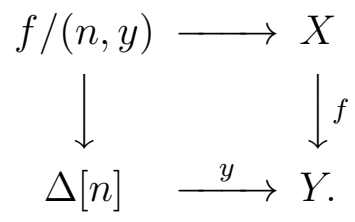

Lemma B ([25]). If for every $u: \Delta^{m} \longrightarrow \Delta^{n}$, and every $y \in Y_{n}$, the induced map from $f /\left(m, u^{*} y\right)$ to $f /(n, y)$ is a homotopy equivalence then for every $(n, y)$ the pullback diagram above is homotopy cartesian.

Lemma 3.4. For every $k \geqslant 0$, the map $f: i_{k}$ S.E $\longrightarrow i_{k}$ S.A sending a string $E$ to the string $A=A_{0} \stackrel{\sim}{\longrightarrow} A_{1} \stackrel{\sim}{\longrightarrow} \cdots \stackrel{\sim}{\longrightarrow} A_{k}$ satisfies the hypothesis of Lemma $B$.

By Lemma B we obtain a homotopy fibration $f /(n, A) \longrightarrow i_{k} \mathbf{S} \cdot \mathscr{E} \longrightarrow i_{k} \mathbf{S} . \mathscr{A}$ for every simplex $A$ of $i_{k} \mathbf{S}$. $\mathscr{A}$. In particular the sequence $f /(0,0) \longrightarrow i_{k} \mathbf{S} . \mathscr{E} \longrightarrow i_{k} \mathbf{S} . \mathscr{A}$ is a homotopy fibration for the unique 0 -simplex. The term $f /(0,0)$ can be identified to the simplicial set $i_{k} \mathbf{S} . \mathscr{E} \prime$ consisting of the strings $E \in i_{k} \mathbf{S} . \mathscr{E}$ such that $A_{j}=0$ and $C_{j} \longrightarrow B_{j}, j \leqslant k$, is an isomorphism. The latter simplicial set is homotopy equivalent 
to $i_{k} \mathbf{S} . \mathscr{A}$ by the sublemma below via the exact equivalence $E \in \mathscr{E} \longrightarrow B \in \mathscr{A}$. The simplicial set $i_{k} \mathbf{S} . \mathscr{A}$ is connected and therefore the sequence

$$
i . \mathbf{S} . \mathscr{A} \stackrel{g}{\longrightarrow} i . \mathbf{S} . \mathscr{E} \stackrel{f}{\longrightarrow} i . \mathbf{S} . \mathscr{A}
$$

with $B \stackrel{g}{\longmapsto} 0 \longmapsto B \rightarrow B$ is a fibration by Lemma 3.3

Finally, consider a morphism of the latter fibration sequence to the trivial product fibration sequence,



The map is a homotopy equivalence on the fibre and on the base, and hence is so on the total spaces. Thus Lemma 3.4 implies the additivity theorem.

Let $C$ and $D$ be two simplicial objects in a category $\mathscr{C}$ and let $\Delta / \Delta^{1}$ denote the category of objects over $\Delta^{1}$ in $\Delta$; the objects are the maps $\Delta^{n} \longrightarrow \Delta^{1}$. For any simplicial object $C$ in $\mathscr{C}$ let $C^{*}$ denote the composed functor

$$
\begin{gathered}
\left(\Delta / \Delta^{1}\right)^{\text {op }} \longrightarrow \Delta^{\text {op }} \stackrel{C}{\longrightarrow} \mathscr{C} \\
\left(\Delta^{n} \longrightarrow \Delta^{1}\right) \longmapsto \Delta^{n} \longmapsto C_{n} .
\end{gathered}
$$

Then a simplicial homotopy of maps from $C$ to $D$ is a natural transformation $C^{*} \longrightarrow$ $D^{*}$ [25, p. 335].

Sublemma. Let $\mathscr{A}$ and $\mathscr{A}^{\prime}$ be two exact categories. Then an isomorphism between two exact functors $f, g: \mathscr{A} \longrightarrow \mathscr{A}^{\prime}$ induces a homotopy between $f_{*}$ and $g_{*}: i_{k}$ S.A $\longrightarrow$ $i_{k} \mathrm{~S} . \mathscr{A}^{\prime}$ for every $k \geqslant 0$. In particular, every exact equivalence $\mathscr{A} \longrightarrow \mathscr{A}^{\prime}$ induces a homotopy equivalence $i_{k} \mathbf{S} . \mathscr{A} \longrightarrow i_{k} \mathbf{S} . \mathscr{A}^{\prime}$.

Proof. The proof is similar to that of [25, 1.4.1].

Proof of Lemma 3.4 To simplify the notation the maps $\operatorname{Ar} \Delta^{m} \longrightarrow \operatorname{Ar} \Delta^{n}$ induced by the maps $u: \Delta^{m} \longrightarrow \Delta^{n}$ we denote by the same letter. We must show that for every $A^{\prime} \in i_{k} \mathbf{S}_{n} \mathscr{A}$ and $u: \Delta^{m} \longrightarrow \Delta^{n}$ in $\Delta$, the map $u_{*}: f /\left(m, u^{*} A^{\prime}\right) \longrightarrow f /\left(n, A^{\prime}\right)$ is a homotopy equivalence. Since there are maps $v: \Delta^{0} \longrightarrow \Delta^{n}$ and $w: \Delta^{0} \longrightarrow \Delta^{m}$ such that $u w=v$, it suffices to consider the special class of maps $\Delta^{0} \longrightarrow \Delta^{n}$. Indeed, if we proved that both $v_{*}$ and $w_{*}$ are homotopy equivalences, then it would follow that $u_{*}$ is a homotopy equivalence, too.

So we must prove the following special case: let $A^{\prime}$ be a $n$-simplex of $i_{k} \mathbf{S}$. $\mathscr{A}$, for some $n$, and let $v_{i}: \Delta^{0} \longrightarrow \Delta^{n}$ be the map taking 0 to $i$. Then for every $i$ the map

$$
v_{i *}: f /(0,0) \longrightarrow f /\left(n, A^{\prime}\right)
$$

is a homotopy equivalence.

A $m$-simplex of $f /\left(n, A^{\prime}\right)$ consists of a $m$-simplex $E$ of $i_{k} \mathbf{S}_{m} \mathscr{E}$ together with a map $u: \operatorname{Ar} \Delta^{m} \longrightarrow \operatorname{Ar} \Delta^{n}$ such that $u^{*} A^{\prime}=E_{(0,0)}$. The map sending $E$ to $E_{(1,1)}$ induces a map $p: f /\left(n, A^{\prime}\right) \longrightarrow i_{k} \mathbf{S}$. A . It will suffice to show that $p$ is a homotopy equivalence. Indeed, $p$ is left inverse to the composed map

$$
i_{k} \mathbf{S} . \mathscr{A} \stackrel{\beta_{*}}{\longrightarrow} f /(0,0) \stackrel{v_{i *}}{\longrightarrow} f /(n, A)
$$


therefore if $p$ is a homotopy equivalence then so is $v_{i *} \beta$ and hence also $v_{i *}$, since the map $\beta$ taking $B$ to $0 \longmapsto B \rightarrow B$ is a homotopy equivalence by the sublemma above. This implies $v_{i *}$ is a homotopy equivalence, too. To prove that $p$ is a homotopy equivalence, it suffices to show that the particular map $v_{n *}$ is a homotopy equivalence, because $p v_{n *} \beta=1$.

We shall construct the homotopy by lifting the simplicial homotopy that contracts $\Delta[n]$ to its last vertex. This simplicial homotopy is given by a map of the composed functors

$$
\begin{gathered}
\left(\Delta / \Delta^{1}\right)^{\text {op }} \longrightarrow \Delta^{\mathrm{op}} \longrightarrow \text { Sets } \\
\left(\Delta^{m} \longrightarrow \Delta^{1}\right) \longmapsto \Delta^{m} \longmapsto \operatorname{Hom}_{\Delta}\left(\Delta^{m}, \Delta^{n}\right)
\end{gathered}
$$

to itself. Precisely, the functor takes $v: \Delta^{m} \longrightarrow \Delta^{1}$ to

$$
\left(u: \Delta^{m} \longrightarrow \Delta^{n}\right) \longmapsto\left(\bar{u}: \Delta^{m} \longrightarrow \Delta^{n}\right)
$$

where $\bar{u}$ is defined as the composite

$$
\Delta^{m} \stackrel{(u, v)}{\longrightarrow} \Delta^{n} \times \Delta^{1} \stackrel{w}{\longrightarrow} \Delta^{n}
$$

and where $w(j, 0)=j$ and $w(j, 1)=n$.

A lifting of this homotopy to one on $f /\left(n, A^{\prime}\right)$ is a map taking $v: \Delta^{m} \longrightarrow \Delta^{1}$ to

$$
(E, u) \longrightarrow(\bar{E}, \bar{u})
$$

with $\bar{E}_{(0,0)}=\bar{u}^{*} A^{\prime}$. We shall depict the elements of $i_{k} \mathbf{S}_{m} \mathscr{E}$ as diagrams

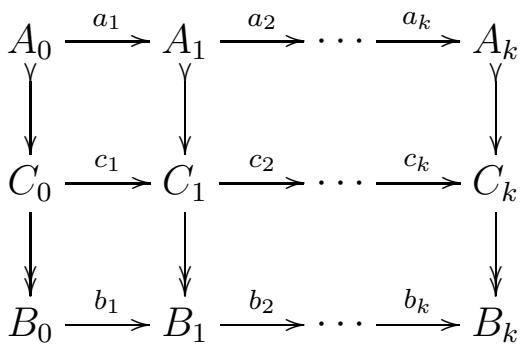

with $A_{i}=u^{*} A_{i}^{\prime}, a_{i}=u^{*}\left(a_{i}^{\prime}: A_{i-1}^{\prime} \longrightarrow A_{i}^{\prime}\right)$, and $\left(a_{i}, c_{i}, b_{i}\right): E_{i-1} \longrightarrow E_{i}$ isomorphisms in $D^{b}\left(S_{m} \mathscr{E}\right), i \leqslant k$. Each vertical map is represented by a commutative diagram

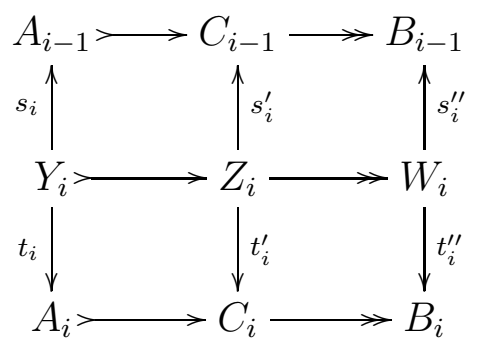

in $C^{b}\left(S_{m} \mathscr{A}\right)$ with the vertical maps quasi-isomorphisms and $t_{i} s_{i}^{-1}=a_{i}$.

Since $u \leqslant \bar{u}$ by construction, it follows that there is a bimorphism $\varphi: u \longrightarrow \bar{u}$. This bimorphism is actually unique, because we deal with maps of posets. This yields a map $\varphi_{A_{i}}^{*}: A_{i} \longrightarrow \bar{A}_{i}$ for every $i$ where $\bar{A}_{i}=\bar{u}^{*} A_{i}^{\prime}$. By assumption, each morphism 
$a_{i}: A_{i-1} \longrightarrow A_{i}$ equals to $u^{*}\left(a_{i}^{\prime}\right)$ where $a_{i}^{\prime}: A_{i-1}^{\prime} \longrightarrow A_{i}^{\prime}$ is an isomorphism in $D^{b}\left(S_{n} \mathscr{A}\right)$ represented by the equivalence class of a diagram

$$
A_{i-1}^{\prime} \stackrel{p_{i}^{\prime}}{\longleftarrow} X_{i}^{\prime} \stackrel{q_{i}^{\prime}}{\longrightarrow} A_{i}^{\prime}
$$

with $p_{i}^{\prime}, q_{i}^{\prime}$ quasi-isomorphisms in $C^{b}\left(S_{n} \mathscr{A}\right)$. Then $a_{i}=q_{i} p_{i}^{-1}$ where $p_{i}=u^{*}\left(p_{i}^{\prime}\right)$ and $q_{i}=u^{*}\left(q_{i}^{\prime}\right)$.

There is a common denominator

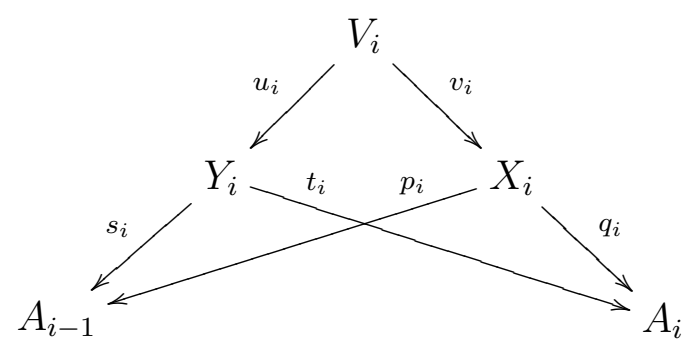

By Lemma 2.2 there exists a complex $U_{i} \in C^{b}\left(S_{m} \mathscr{A}\right)$ and a commutative diagram

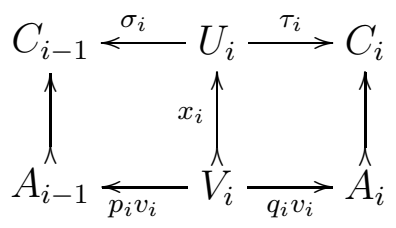

representing the same morphism in $D^{b}\left(\left[S_{m} \mathscr{A}\right]^{\Delta^{1}}\right)$

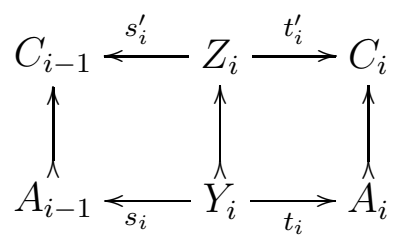

Let $\bar{a}_{i}: \bar{A}_{i-1} \longrightarrow \bar{A}_{i}$ be the map represented by the equivalence class of the diagram

$$
\bar{A}_{i-1} \stackrel{\bar{p}_{i}}{\longleftarrow} \bar{X}_{i}=\bar{u}^{*} X_{i}^{\prime} \stackrel{\bar{q}_{i}}{\longrightarrow} \bar{A}_{i}
$$

with $\bar{p}_{i}=\bar{u}^{*}\left(p_{i}^{\prime}\right), \bar{q}_{i}=\bar{u}^{*}\left(q_{i}^{\prime}\right)$. Then $\bar{a}_{i}$ is an isomorphism since $a_{i}^{\prime}$ is so.

We obtain a commutative diagram

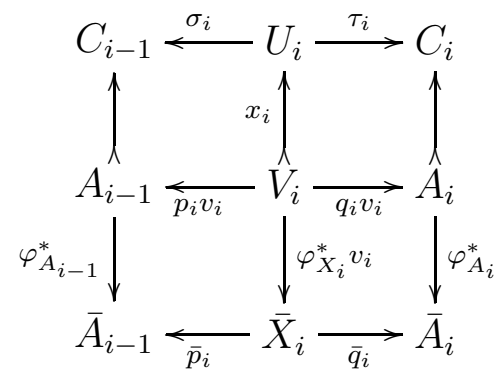

giving an isomorphism $\left(\bar{a}_{i}, a_{i}, c_{i}\right)$ in $D^{b}\left(\widetilde{\left[S_{m} \mathscr{A}\right.}\right]\ulcorner)$. 
Sublemma. The map $\left(\bar{a}_{i}, a_{i}, c_{i}\right)$ represented by diagram (5) is well defined that is it does not depend on:

(1) the choice of a common denominator (3);

(2) the choice of a representative for $\left(a_{i}, c_{i}, b_{i}\right): E_{i-1} \longrightarrow E_{i}$;

(3) the choice of a representative for $a_{i}^{\prime}: A_{i-1}^{\prime} \longrightarrow A_{i}^{\prime}$.

Proof. Let us check (1). Suppose we are given the following diagram.

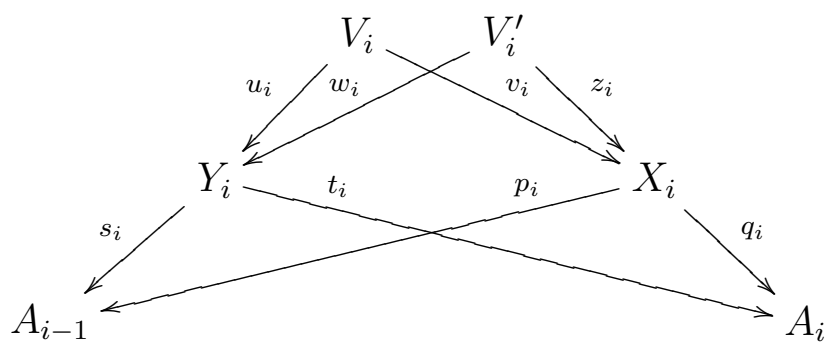

We have to show that

(6)

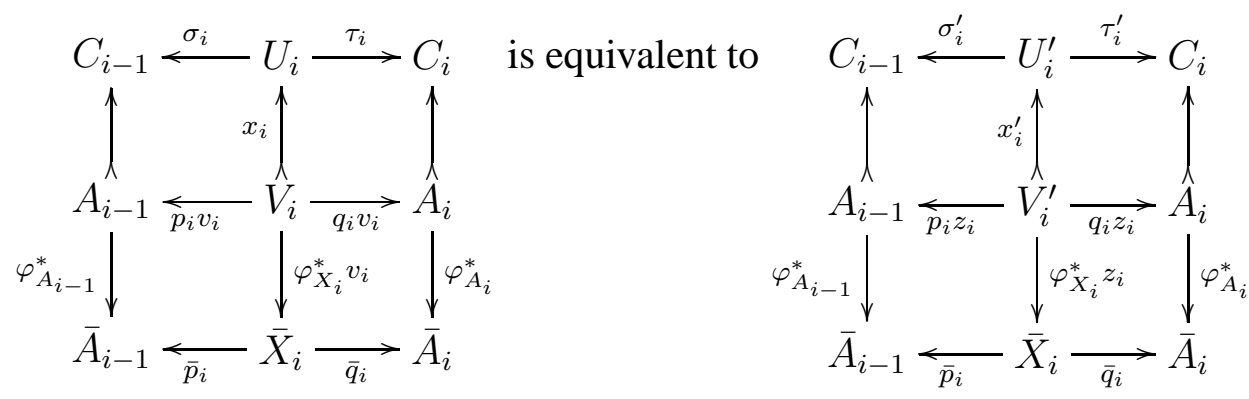

By Lemma 2.2 there is a common denominator



Fix homotopies $\left(e^{n}, g^{n}\right):\left(V_{i}^{\prime \prime n} \stackrel{x_{i}^{\prime \prime}}{\longrightarrow} U_{i}^{\prime \prime n}\right) \longrightarrow\left(A_{i-1}^{n-1} \longrightarrow C_{i-1}^{n-1}\right)$ and $\left(h^{n}, m^{n}\right)$ : $\left(V_{i}^{\prime \prime n} \stackrel{x_{i}^{\prime \prime}}{\longrightarrow} U_{i}^{\prime \prime n}\right) \longrightarrow\left(A_{i}^{n-1} \longrightarrow C_{i}^{n-1}\right)$ for $\left(p_{i} v_{i} c_{i}, \sigma_{i} l_{i}\right) \sim\left(p_{i} z_{i} d_{i}, \sigma_{i}^{\prime} f_{i}\right)$ and $\left(q_{i} v_{i} c_{i}, \tau_{i} l_{i}\right) \sim$ 
$\left(q_{i} z_{i} d_{i}, \tau_{i}^{\prime} f_{i}\right)$ respectively. The latter diagram fits into the diagram

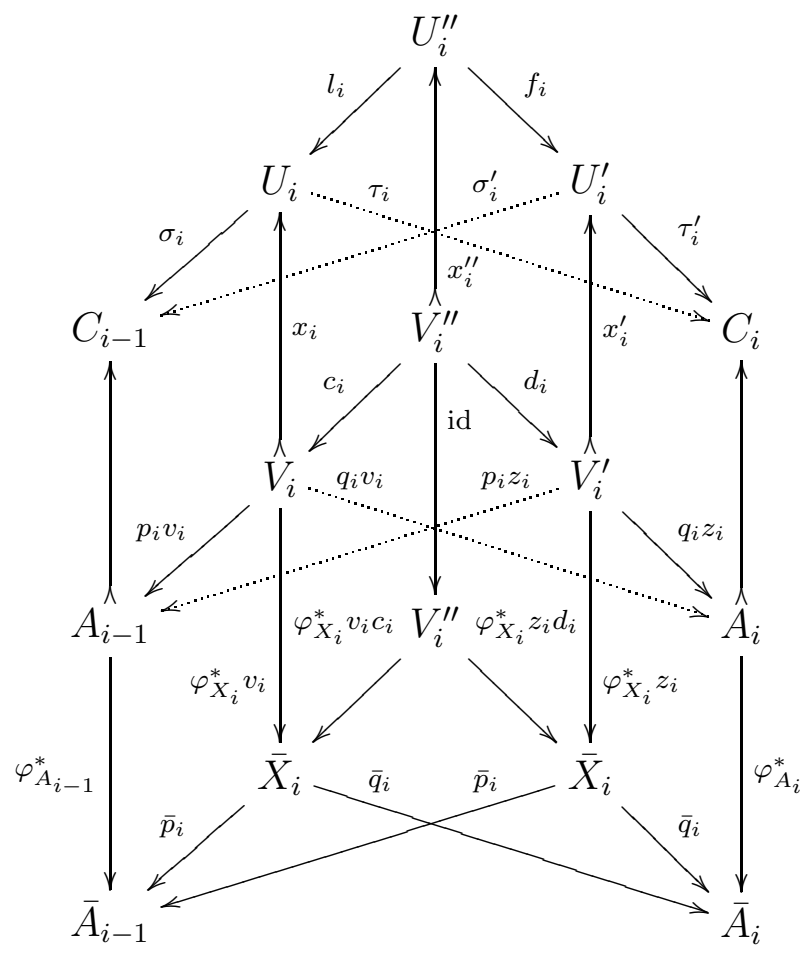

We want to show that this diagram is a common denominator for (6). Put $k^{n}=\varphi_{A_{i-1}}^{* n-1} \circ$ $e^{n}: V^{\prime \prime n} \longrightarrow \bar{A}_{i-1}^{n-1}$. Then

$$
\begin{gathered}
\bar{p}_{i} \varphi_{X_{i}}^{*} v_{i} c_{i}-\bar{p}_{i} \varphi_{X_{i}}^{*} z_{i} d_{i}=\varphi_{A_{i-1}}^{*} p_{i} v_{i} c_{i}-\varphi_{A_{i-1}}^{*} p_{i} z_{i} d_{i}= \\
=\varphi_{A_{i-1}}^{*} e \partial+\varphi_{A_{i-1}}^{*} \partial e=k \partial+\partial \varphi_{A_{i-1}}^{*}=k \partial+\partial k .
\end{gathered}
$$

This shows that $\left(\sigma_{i} l_{i}, p_{i} v_{i} c_{i}, \bar{p}_{i} \varphi_{X_{i}}^{*} v_{i} c_{i}\right) \stackrel{(g, e, k)}{\sim}\left(\sigma_{i}^{\prime} f_{i}, p_{i} z_{i} d_{i}, \bar{p}_{i} \varphi_{X_{i}}^{*} z_{i} d_{i}\right)$. A homotopy between $\left(\tau_{i} l_{i}, q_{i} v_{i} c_{i}, \bar{q}_{i} \varphi_{X_{i}}^{*} v_{i} c_{i}\right)$ and $\left(\tau_{i}^{\prime} f_{i}, q_{i} z_{i} d_{i}, \bar{q}_{i} \varphi_{X_{i}}^{*} z_{i} d_{i}\right)$ is similarly checked.

Let us check (2). Suppose

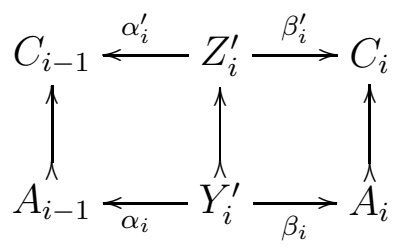

is equivalent to (4). There is a homotopy commutative diagram

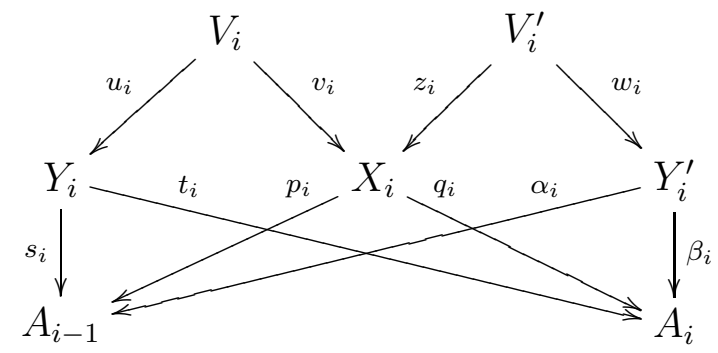


We have to show that

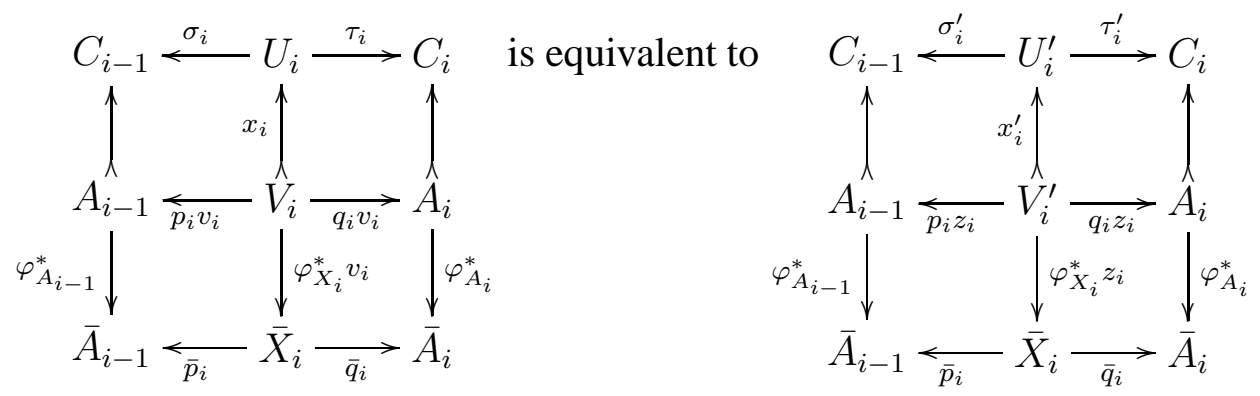

It follows from Lemma 2.2 and our assumption that
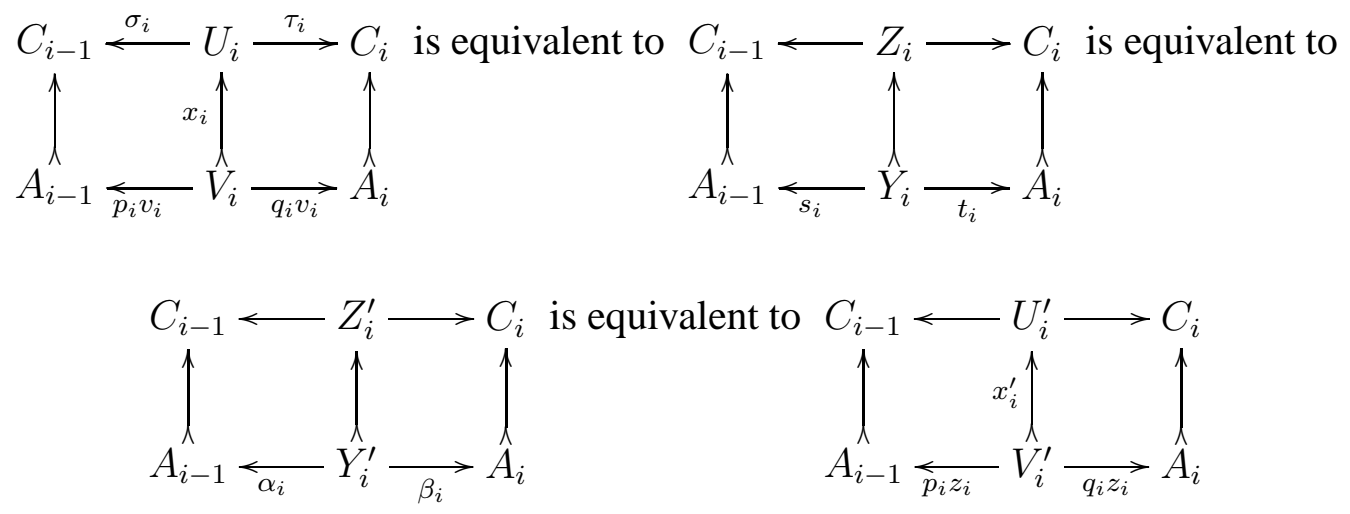

One can now construct the diagram (7) yielding a common denominator for (8). This implies (2). It remains to check (3).

Let $A_{i-1}^{\prime} \stackrel{r_{i}^{\prime}}{\longleftarrow} W_{i}^{\prime} \stackrel{n_{i}^{\prime}}{\longrightarrow} A_{i}^{\prime}$ be another representative for $a_{i}^{\prime}$. There is a homotopy commutative diagram

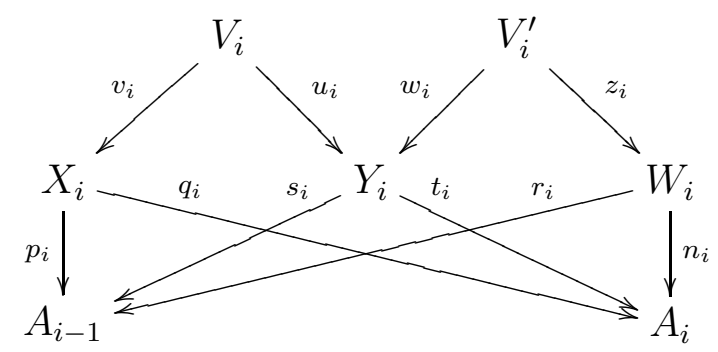

with $r_{i}=u^{*}\left(r_{i}^{\prime}\right), n_{i}=u^{*}\left(n_{i}^{\prime}\right)$. We have to show that

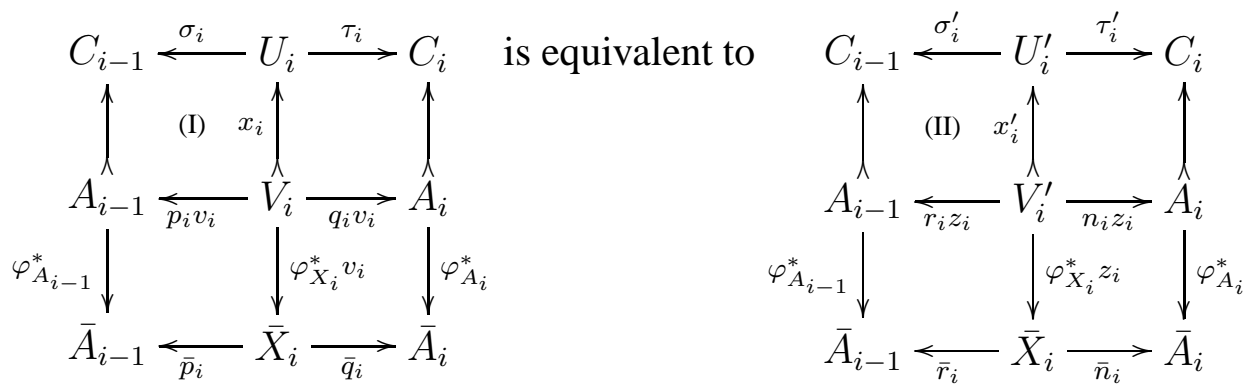


There are common denominators in $C^{b}\left(S_{n} \mathscr{A}\right)$ and in $C^{b}\left(S_{m} \mathscr{A}\right)$, respectively.

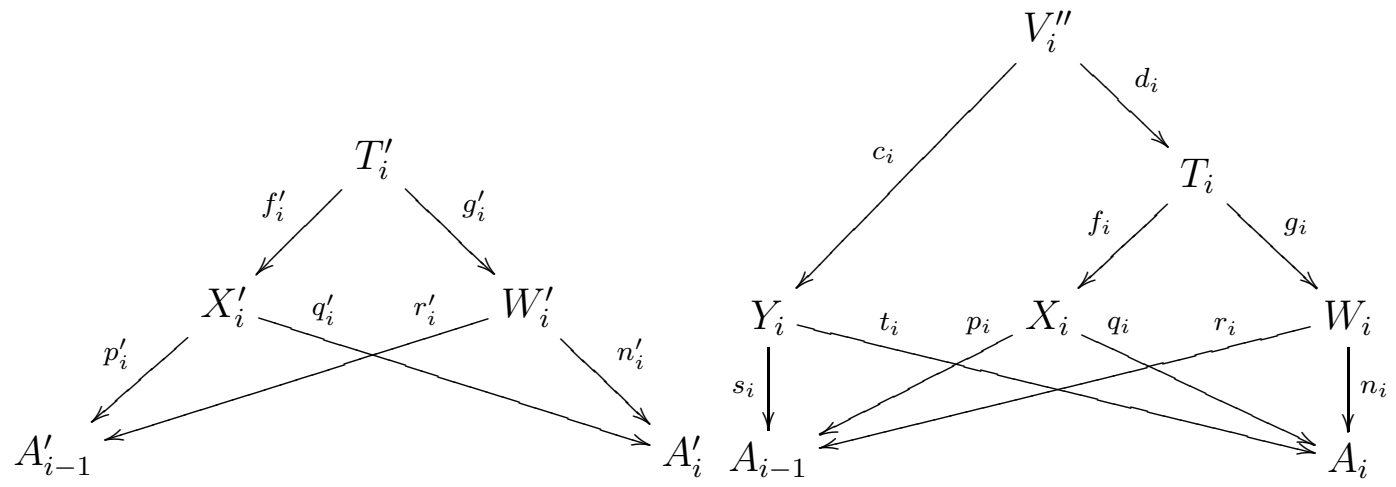

It follows from (1) that



Since $\varphi_{X_{i}}^{*} f_{i}=\bar{f}_{i} \varphi_{T_{i}}^{*}$ and $\varphi_{W_{i}}^{*} g_{i}=\bar{g}_{i} \varphi_{T_{i}}^{*}$, it follows that
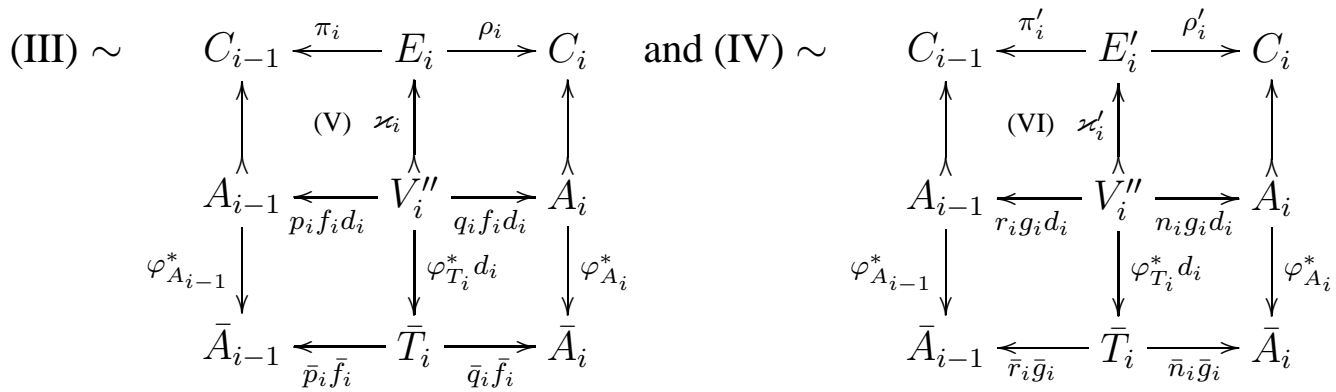

Fix homotopies $p_{i}^{\prime} f_{i}^{\prime} \stackrel{k^{\prime}}{\sim} r_{i}^{\prime} g_{i}^{\prime}, n_{i}^{\prime} g_{i}^{\prime} \stackrel{e^{\prime}}{\sim} q_{i}^{\prime} f_{i}^{\prime}$. Then $(k, e)=\left(u^{*}\left(k^{\prime}\right), u^{*}\left(e^{\prime}\right)\right)$ and $(\bar{k}, \bar{e})=$ $\left(\bar{u}^{*}\left(k^{\prime}\right), \bar{u}^{*}\left(e^{\prime}\right)\right)$ give homotopies $p_{i} f_{i} \stackrel{k}{\sim} r_{i} g_{i}, n_{i} g_{i} \stackrel{e}{\sim} q_{i} f_{i}$ and $\bar{p}_{i} \bar{f}_{i} \stackrel{\bar{k}}{\sim} \bar{r}_{i} \bar{g}_{i}, \bar{n}_{i} \bar{g}_{i} \stackrel{\bar{e}}{\sim} \bar{q}_{i} \bar{f}_{i}$. The 
proof of Lemma 2.2 shows that $(\mathrm{V})$ can be embedded into the diagram

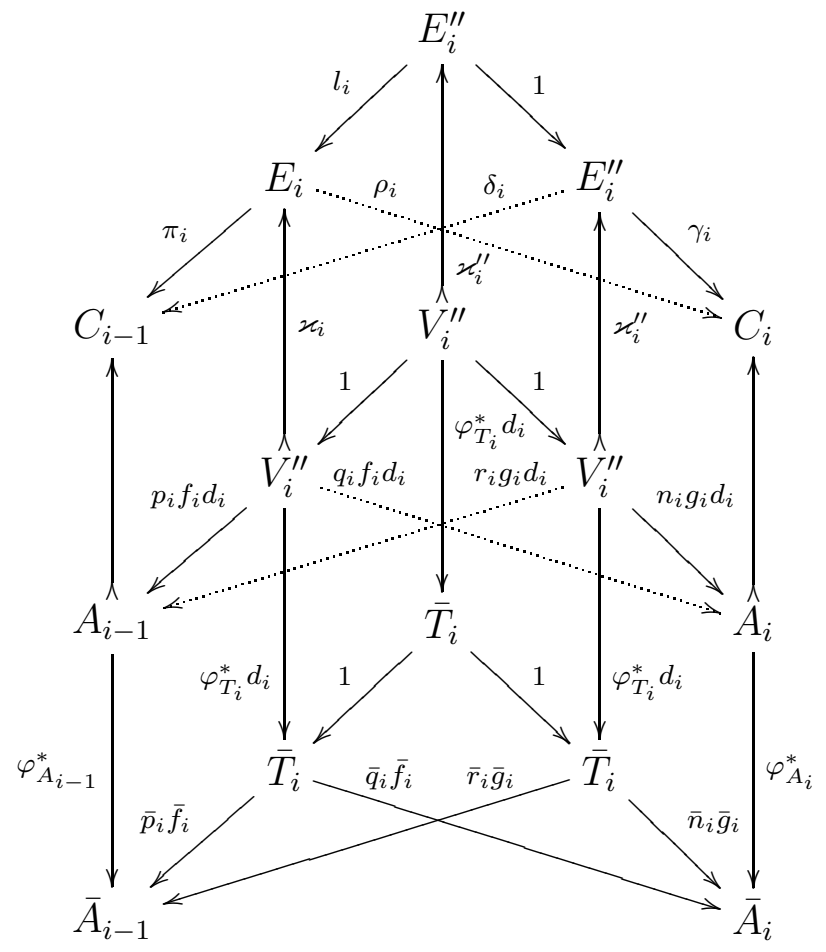

in such a way that the upper "roof" is a common denominator with homotopies $\left(p_{i} f_{i} d_{i}, \pi_{i} l_{i}\right) \stackrel{\left(k d_{i}, h\right)}{\sim}$ $\left(r_{i} g_{i} d_{i}, \delta_{i}\right)$ and $\left(n_{i} g_{i} d_{i}, \gamma_{i}\right) \stackrel{\left(e d_{i}, \ell\right)}{\sim}\left(q_{i} f_{i} d_{i}, \rho_{i} l_{i}\right)$. We have homotopies $\left(\bar{p}_{i} \bar{f}_{i}, p_{i} f_{i} d_{i}, \pi_{i} l_{i}\right) \stackrel{\left(\bar{k}, k d_{i}, h\right)}{\sim}$ $\left(\bar{r}_{i} \bar{g}_{i}, r_{i} g_{i} d_{i}, \delta_{i}\right)$ and $\left(\bar{n}_{i} \bar{g}_{i}, n_{i} g_{i} d_{i}, \gamma_{i}\right) \stackrel{\left(\bar{e}, e d_{i}, \ell\right)}{\sim}\left(\bar{q}_{i} \bar{f}_{i}, q_{i} f_{i} d_{i}, \rho_{i} l_{i}\right)$ showing that

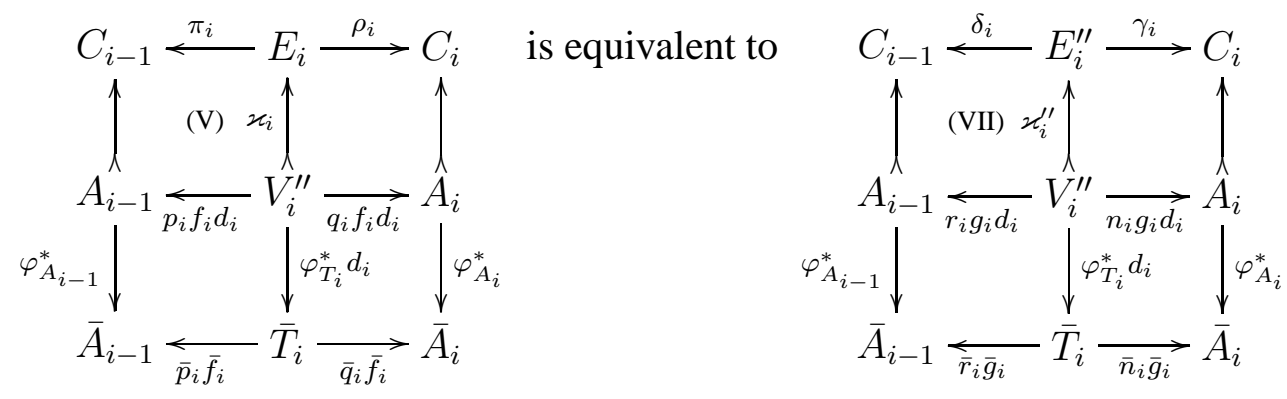

The proof of $(1)$ shows that $(\mathrm{VI}) \sim(\mathrm{VII})$, hence $(\mathrm{I}) \sim(\mathrm{III}) \sim(\mathrm{V}) \sim(\mathrm{VII}) \sim(\mathrm{VI}) \sim$ $(\mathrm{IV}) \sim(\mathrm{II})$ as required.

For every $i \leqslant k$, we can construct a pushout diagram in $C^{b}\left(S_{m} \mathscr{A}\right)$

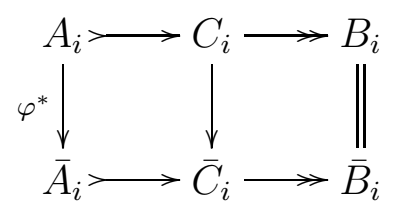


The diagrams (5) give a string of isomorphisms in $D^{b}\left(\left[\widetilde{S_{m} \mathscr{A}}\right]\ulcorner)\right.$

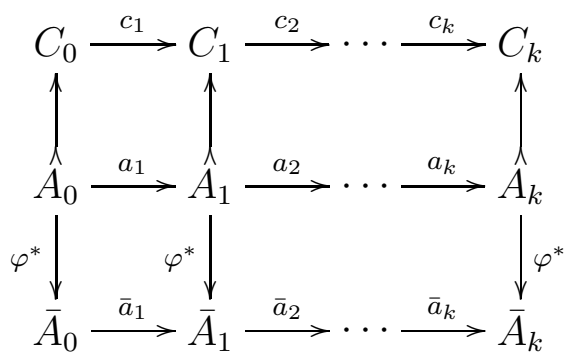

By Corollary 2.3 one can fit it into a string of isomorphisms in $\left.D^{b}\left(\widetilde{\left[S_{m} \mathscr{A}\right.}\right]^{\square}\right)$

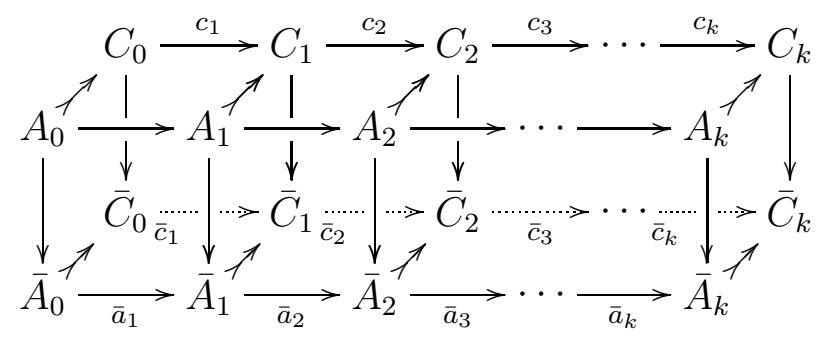

Finally, we consider the diagram constructed as above

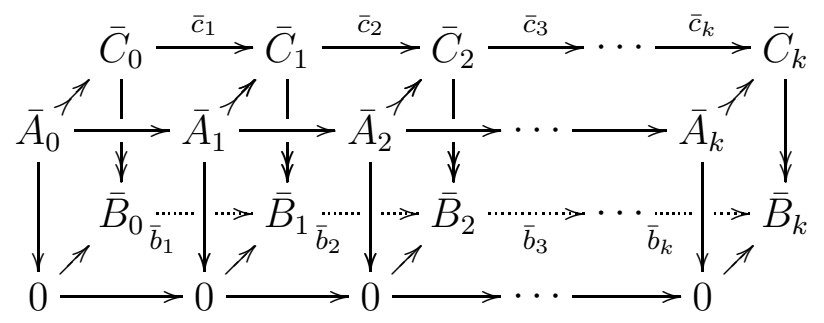

Construction of the simplex $(\bar{E}, \bar{u})$ is completed. It is given by the diagram

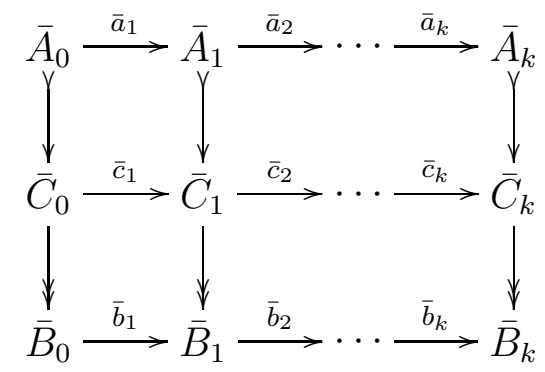

with $\bar{A}_{i}=\bar{u}^{*} A_{i}^{\prime}$ and $\left(\bar{a}_{i}, \bar{c}_{i}, \bar{b}_{i}\right): \bar{E}_{i-1} \longrightarrow \bar{E}_{i}$ isomorphisms in $D^{b}\left(S_{m} \mathscr{E}\right), i \leqslant k$.

We have to verify that the construction of the simplex $(\bar{E}, \bar{u})$ is compatible with the structure maps of the category $\Delta / \Delta^{1}$; that is, if we replace $\Delta^{m}$ by $\Delta^{m^{\prime}}$ throughout, by means of some map $\Delta^{m^{\prime}} \longrightarrow \Delta^{m}$, then the strucure map in $i_{k}$ S.E्E induced by $\Delta^{m^{\prime}} \longrightarrow$ $\Delta^{m}$ takes one simplex to the other.

To see this, we repeat the steps of the construction. The first step was the definition of the map $\varphi^{*}: A \longrightarrow \bar{A}$. The definition is compatible with structure maps because the bimorphism $\varphi: u \longrightarrow \bar{u}$ is uniquely defined. 
The second step was the choice of pushout diagrams (9). But this choice is made in $\mathscr{C}=C^{b}(\mathscr{A})$, and an element of $S_{m} \mathscr{C}$ is a certain diagram in $\mathscr{C}$ on which the simplicial structure maps in $S . \mathscr{C}$ operate by omission and/or reduplicating of data. So again there is the required compatibility.

The third step was construction of the isomorphisms $\left(\bar{a}_{i}, \bar{c}_{i}, \bar{b}_{i}\right): \bar{E}_{i-1} \longrightarrow \bar{E}_{i}$. The desired compatibility follows from the fact that the maps $\left(\bar{a}_{i}, a_{i}, c_{i}\right)$ represented by diagrams (5) are well defined.

With an extra care one can arrange the choices so that the homotopy starts from the identity map (namely if $A \longrightarrow \bar{A}$ is an identity map we insist that $C \longrightarrow \bar{C}$ is also an identity map); and that the image of $v_{n *} j_{*}$ is fixed under the homotopy (namely if $\bar{A}=0$ we insist that $\bar{C} \longrightarrow \bar{B}$ is the identity map on $\bar{B}$ ). We have now constructed the desired homotopy. This completes the proof.

Now we discuss some immediate consequences of the additivity theorem. Let $\mathscr{A}$ and $\mathscr{A}^{\prime}$ be two exact categories. By an exact sequence of exact functors $\mathscr{A} \longrightarrow \mathscr{A}^{\prime}$ is meant a sequence of natural transformations $F^{\prime} \longrightarrow F \longrightarrow F^{\prime \prime}$ such that for every $A \in \mathscr{A}$ the sequence $F^{\prime}(A) \longrightarrow F(A) \longrightarrow F^{\prime \prime}(A)$ is exact in $\mathscr{A}^{\prime}$.

If $\mathscr{A}^{\prime}, \mathscr{A}^{\prime \prime}$ are fully exact subcategories of an exact category $\mathscr{A}$ by $\mathscr{E}\left(\mathscr{A}^{\prime}, \mathscr{A}, \mathscr{A}^{\prime \prime}\right)$ denote the exact subcategory of the exact extension category $\mathscr{E}=\mathscr{E}(\mathscr{A})$ with the source and target entries in $\mathscr{A}^{\prime}$ and $\mathscr{A}^{\prime \prime}$ respectively.

Proposition 3.5 (Equivalent formulations of the additivity theorem). Each of the following conditions is equivalent to the additivity theorem (Theorem 3.1).

(1) The following projection is a homotopy equivalence,

$$
i . \mathbf{S} . \mathscr{E}\left(\mathscr{A}^{\prime}, \mathscr{A}, \mathscr{A}^{\prime \prime}\right) \longrightarrow i . \mathbf{S} . \mathscr{A}^{\prime} \times i . \mathbf{S} . \mathscr{A}^{\prime \prime}, \quad A \longmapsto C \rightarrow B \longmapsto(A, B) .
$$

(2) The following two maps are homotopic,

$$
i . \text { S. } \mathscr{E} \longrightarrow i . \text { S.A }, \quad A \longmapsto C \rightarrow B \longmapsto C \text {, respectively } A \oplus B .
$$

(3) If $F^{\prime} \longrightarrow F \longrightarrow F^{\prime \prime}$ is an exact sequence of exact functors $\mathscr{A} \longrightarrow \mathscr{A}^{\prime}$ then there exists a homotopy

$$
|i . \mathbf{S} . F| \simeq\left|i . \mathbf{S} . F^{\prime}\right| \vee\left|i . \mathbf{S} . F^{\prime \prime}\right| .
$$

Proof. The proof is similar to that of [25, 1.3.2].

If $F: \mathscr{A} \longrightarrow \mathscr{C}$ and $G: \mathscr{B} \longrightarrow \mathscr{C}$ be two arbitrary functors with common codomain, the fibre product $\prod(F, G)$ is defined as the category of triples

$$
(A, c, B), A \in \mathscr{A}, B \in \mathscr{B}, c: F(A) \longrightarrow G(B) \text { is an isomorphism. }
$$

This is equivalent to the pullback category in special cases, for example if one of $F$ and $G$ is a retraction, but not in general. It follows from [24 p. 180] that if $F$ and $G$ are exact functors then $\prod(F, G)$ is an exact category in a natural way, and the projections to $\mathscr{A}$ and $\mathscr{B}$ are exact functors. This is directly extended to the definition of the fibred product for simplicial exact functors of simplicial exact categories.

Let $\mathscr{B}$ be an exact category and $P(S . \mathscr{B})$ be the path space for the simplicial exact category $S . \mathscr{B}$. One has the simplicial map $\partial_{0}: P(S . \mathscr{B}) \longrightarrow S . \mathscr{B}$. Let $F: \mathscr{A} \longrightarrow \mathscr{B}$ be 
an exact functor with $\mathscr{A}$ an exact category. Denote by $S .(\mathscr{A} \longrightarrow \mathscr{B})$ the fibred product of the diagram

$$
S . \mathscr{A} \stackrel{F}{\longrightarrow} S . \mathscr{B} \stackrel{\partial_{0}}{\longleftarrow} P(S . \mathscr{B}) .
$$

Each $S_{n}(\mathscr{A} \longrightarrow \mathscr{B}), n \geqslant 0$, consists of the triples

$$
(A, c, B), A \in S_{n} \mathscr{A}, B \in S_{n+1} \mathscr{B}, c: F A \stackrel{\sim}{\longrightarrow} \partial_{0} B .
$$

It is an exact category by above.

Considering $\mathscr{B}$ as a simplicial category in a trivial way we have an inclusion $\mathscr{B} \longrightarrow$ $P(S . \mathscr{B})$ whose composition with the projection to $S . \mathscr{B}$ is trivial. Lifting this inclusion to $S .(\mathscr{A} \longrightarrow \mathscr{B})$, and combining with the other projection, we then obtain a sequence

$$
D^{b}(\mathscr{B}) \longrightarrow \mathrm{S} .(\mathscr{A} \longrightarrow \mathscr{B}) \longrightarrow \mathrm{S} . \mathscr{A}
$$

in which S. $(\mathscr{A} \longrightarrow \mathscr{B})=D^{b}(S .(\mathscr{A} \longrightarrow \mathscr{B}))$ and the composed map is trivial.

Proposition 3.6. The sequence

$$
i . \text { S. } \mathscr{B} \longrightarrow i . \text { S.S. }(\mathscr{A} \longrightarrow \mathscr{B}) \longrightarrow i . \text { S.S.A },
$$

in which S.S. $(\mathscr{A} \longrightarrow \mathscr{B})=D^{b}(S .(S . \mathscr{A} \longrightarrow S . \mathscr{B}))$ is a fibration up to homotopy.

Proof. The proof is similar to that of [25, 1.5.5].

Similarly, there is a sequence

$$
i . \text { S. } \mathscr{B} \longrightarrow P(i . \text { S.S. } \mathscr{B}) \longrightarrow i . \text { S.S. } \mathscr{B}
$$

where the " $P$ " refers to the first $S$-direction, say. As a special case of the preceding proposition this sequence is a fibration up to homotopy.

Thus $\mid i$.S. $\mathscr{B}|\longrightarrow \Omega| i$.S.S. $\mathscr{B} \mid$ is a homotopy equivalence and more generally, in view of Lemma 3.2 also the map $\left|i . \mathbf{S} .^{n} \mathscr{B}\right| \longrightarrow \Omega\left|i . \mathbf{S} .^{n+1} \mathscr{B}\right|$ for every $n \geqslant 1$, proving the postponed claim that the spectrum $n \longmapsto\left|i . \mathbf{S} .^{n} \mathscr{B}\right|$ is a $\Omega$-spectrum beyond the first term.

Corollary 3.7. Suppose we are given a sequence $\mathscr{A} \longrightarrow \mathscr{B} \longrightarrow \mathscr{C}$ of exact functors between exact categories. Then the square

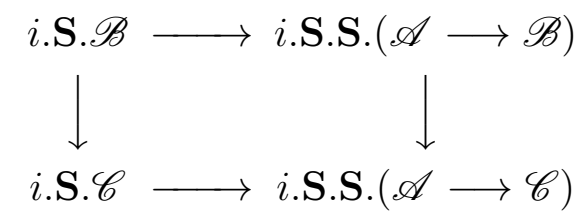

is homotopy cartesian.

Proof. See [25, 1.5.6].

Corollary 3.8. The following two assertions are valid.

(1) To an exact functor there is associated a fibration

$$
i . \mathbf{S} . \mathscr{B} \longrightarrow i . \mathbf{S} . \mathscr{C} \longrightarrow i . \mathbf{S} . \mathbf{S} .(\mathscr{B} \longrightarrow \mathscr{C}) \text {. }
$$

(2) If $\mathscr{C}$ is a retract of $\mathscr{B}$ (by exact functors) there is a splitting

$$
i . \text { S. } \mathscr{B} \simeq i . \text { S. } \mathscr{C} \times i . \text { S.S. }(\mathscr{C} \longrightarrow \mathscr{B}) .
$$

Proof. [25, 1.5.7, 1.5.8]. 
By a nice complicial biWaldhausen category $\mathscr{C}$ formed from the category of complexes $C^{b}(\mathscr{B})$ with $\mathscr{B}$ an abelian category will be meant a complicial biWaldhausen category in the sense of Thomason [22] which is closed under the formation of canonical homotopy pushouts and canonical homotopy pullbacks. For example, let $\mathscr{A}$ be an exact category and $\mathscr{A} \longrightarrow \mathscr{B}$ be the Gabriel-Quillen embedding [22, Appendix A]. Then $C^{b}(\mathscr{A})$ is a nice complicial biWaldhausen category formed from $C^{b}(\mathscr{B})$.

It is directly verified that for any $n$ the category $S_{n} \mathscr{C}$ is a nice complicial biWaldhausen category which is formed from the category of complexes $C^{b}\left(\mathscr{B}^{\operatorname{Ar} \Delta^{n}}\right)$ with $\mathscr{B}^{\operatorname{Ar} \Delta^{n}}$ the abelian functor category $\operatorname{Hom}\left(\operatorname{Ar} \Delta^{n}, \mathscr{B}\right)$. The relevant subcategories of bifibrations and weak equivalences are defined componentwise. In a similar way, given a small category $I$ the diagram category $\mathscr{C}^{I}$ is a nice complicial biWaldhausen category. There results a simplicial nice complicial biWaldhausen category

$$
\text { S. : } \Delta^{n} \longmapsto S_{n} \mathscr{C} \text {. }
$$

Let $w^{-1} \mathscr{C}$ denote the derived category obtained from $\mathscr{C}$ by inverting weak equivalences. It is canonically triangulated and the homotopy category $\mathscr{C} / \simeq$ admits both left and right calculus of fractions [22, p. 269]. One obtains the following bisimplicial object

$$
\text { i.S. : } \Delta^{m} \times \Delta^{n} \longmapsto i_{m} w^{-1} S_{n} \mathscr{C} \text {. }
$$

Denote by $\mathscr{E}(\mathscr{C})$ the extension category of $\mathscr{C}$. Then the proof of the following statement is similar to complexes (all tricks of paragraph 2.3 are also valid for this case).

Corollary 3.9. Let $\mathscr{C}$ be a nice complicial biWaldhausen category. Then the map

$$
i . \mathbf{S} . \mathscr{E}(\mathscr{C}) \stackrel{\left(s_{*}, q_{*}\right)}{\longrightarrow} i . \mathbf{S} . \mathscr{C} \times i . \mathbf{S} . \mathscr{C}
$$

is a homotopy equivalence.

\section{DÉRIVATEURS ASSOCIATED TO COMPLICIAL BIWALDHAUSEN CATEGORIES}

In this section we show that the additivity theorem is valid for dérivateurs associated to nice complicial biWaldhausen categories. Logically, one should now read Addendum, and then return to this section.

Let $\mathscr{C}$ be a nice complicial biWaldhausen category. One of the most important for applications dérivateurs (of the domain $\mathcal{D}$ irf) is given by the hyperfunctor

$$
\mathrm{D} \mathscr{C}: I \in \mathcal{D} \text { irf } w^{-1} \mathscr{C}^{I}
$$

with $w^{-1} \mathscr{C}^{I}$ the derived category of the nice complicial biWaldhausen diagram category $\mathscr{C}^{I}$ [1, 12]. If $\mathscr{C}=C^{b}(\mathscr{A})$ with $\mathscr{A}$ an exact category, the corresponding dérivateur is denoted by $\mathbf{D}^{b}(\mathscr{A})$.

Definition. A left pointed dérivateur $\mathbf{D}$ of the domain $\mathcal{O} r d$ is represented by a nice complicial biWaldhausen category $\mathscr{C}$ if there is a right exact equivalence $F: \mathbf{D} \mathscr{C} \longrightarrow \mathbf{D}$. This equivalence induces a homotopy equivalence of bisimplicial sets $F: i . S . \mathrm{D} \mathscr{C} \longrightarrow$ i.S.D. If $\mathscr{C}=C^{b}(\mathscr{A})$ with $\mathscr{A}$ an exact category, we shall say that $\mathbf{D}$ is represented by $\mathscr{A}$.

Lemma 4.1. The inclusion $F_{n} \mathscr{C} \longrightarrow \mathscr{C}^{\Delta^{n}}$ induces an equivalence of derived categories $w^{-1} F_{n} \mathscr{C} \stackrel{\sim}{\longrightarrow} w^{-1} \mathscr{C}^{\Delta^{n}}$. 
Proof. Since a map in $F_{n} \mathscr{C}$ is a weak equivalence if and only if it is so in $\mathscr{C}^{\Delta^{n}}$, it suffices to show that given an object $A=A_{0} \rightarrow \cdots \rightarrow A_{n}$ in $\mathscr{C}^{\Delta^{n}}$ there is a quasi-isomorphism from an object in $F_{n} \mathscr{C}$ to $A$. Let us consider the following diagram in $\mathscr{C}$.

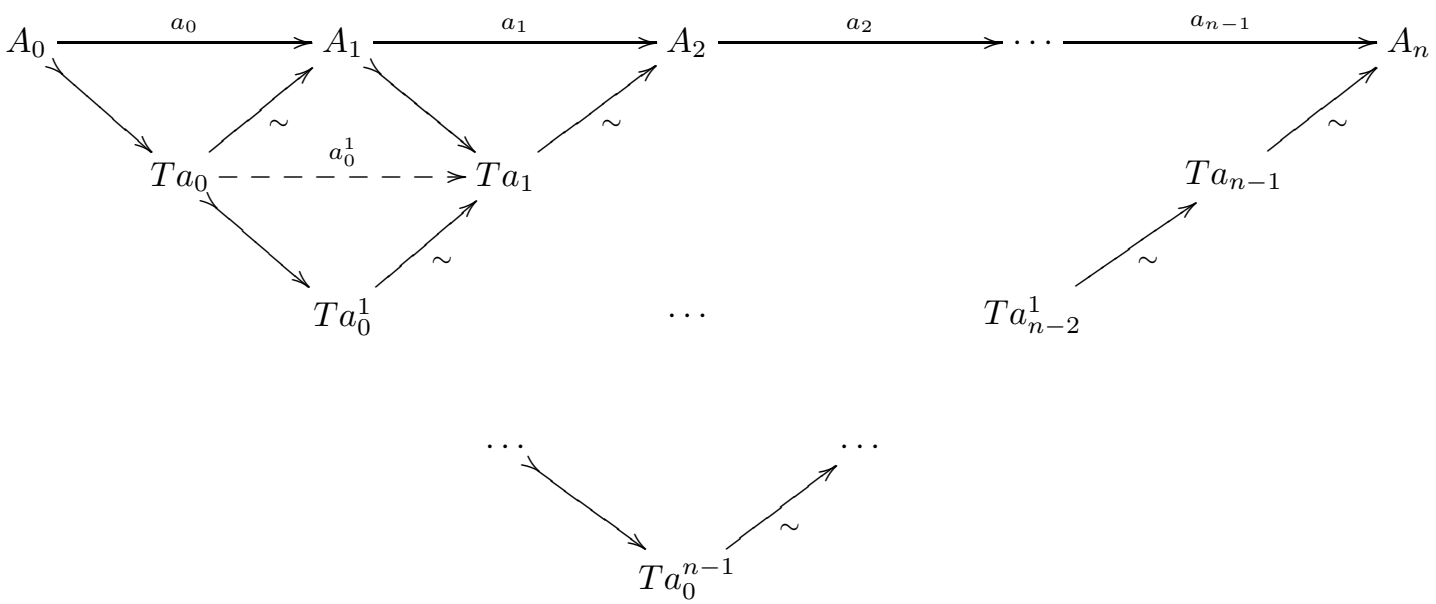

Here $T(-)$ stands for the cylinder object of a morphism, the arrows labelled with " $\sim$ " are weak equivalences, and all the squares of the diagram are commutative. This diagram yields a weak equivalence from the object $A_{0} \longmapsto T a_{0} \longmapsto \cdots \longmapsto T a_{0}^{n-1}$ of $F_{n} \mathscr{C}$ to the object $A_{0} \rightarrow \cdots \rightarrow A_{n}$ of $\mathscr{C}^{\Delta^{n}}$, whence the assertion.

Lemma 4.2. If $\mathrm{D}$ is a dérivateur represented by a nice complicial biWaldhausen category $\mathscr{C}$, then $\mathbf{S}_{n} \mathbf{D}$ is represented by the nice complicial biWaldhausen category $S_{n} \mathscr{C}$ for all $n$.

Proof. The image of each cocartesian square in $\mathscr{C}$ with two parallel arrows cofibrations is cocartesian in $\mathbf{D} \mathscr{C} \square$. This yields a right exact morphism $\mathbf{D} S_{n} \mathscr{C} \longrightarrow \mathbf{S}_{n} \mathbf{D} \mathscr{C}$. Consider the commutative diagram of left pointed dérivateurs

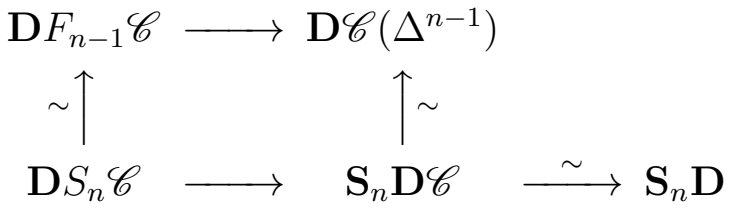

in which the morphisms marked with " $\sim$ " are right exact equivalences. The equivalence on the left is induced by the equivalence $S_{n} \mathscr{C} \longrightarrow F_{n-1} \mathscr{C}$ and the equivalence on the right is a consequence of [6, 3.1].

The upper arrow is an equivalence by Lemma 4.1 We see from the commutative diagram above that $\mathbf{D} S_{n} \mathscr{C} \longrightarrow \mathbf{S}_{n} \mathbf{D} \mathscr{C}$ is a right exact equivalence. Therefore the composed map $\mathbf{D} S_{n} \mathscr{C} \longrightarrow \mathbf{S}_{n} \mathbf{D}$ produces a Waldhausen model for $\mathbf{S}_{n} \mathbf{D}$.

Corollary 4.3. A natural map of bisimplicial sets $i . S . \mathscr{C} \longrightarrow i . S . D \mathscr{C}$ is a homotopy equivalence.

Proof. By the proof of the preceding lemma the functor $w^{-1} S_{n} \mathscr{C} \longrightarrow S_{n} \mathbf{D} \mathscr{C}$ is an equivalence of categories for every $n$. Lemma 3.2 implies the claim.

Let $\mathscr{E}$ denote the extension category of $\mathscr{C}$.

Corollary 4.4. If $\mathbf{D}$ has a Waldhausen model then so does $\mathbf{E}=\mathbf{E}(\mathbf{D})$. 
Proof. It is enough to consider the commutative diagram



in which the left arrow is a right exact equivalence by the exact equivalence of $S_{2} \mathscr{C}$ and $\mathscr{E}$, the upper arrow is a right exact equivalence by the preceding lemma, and the right arrow is a right exact equivalence by [6, 6.2].

There are three natural right exact maps $s, t, q: \mathbf{E} \longrightarrow \mathbf{D}$ taking $E$ to $E_{(0,0)}, E_{(0,1)}$ and $E_{(1,1)}$ respectively. The following result states that the additivity theorem holds for dérivateurs represented by nice complicial biWaldhausen categories.

Theorem 4.5. Let $\mathrm{D}$ be a dérivateur represented by a nice complicial biWaldhausen category. Then the map

$$
i . S . \mathbf{E} \stackrel{\left(s_{*}, q_{*}\right)}{\longrightarrow} i . S . \mathbf{D} \times i . S . \mathbf{D}
$$

is a homotopy equivalence.

Proof. Let $\mathrm{D} \mathscr{C} \stackrel{\sim}{\longrightarrow} \mathrm{D}$ be a Waldhausen model for $\mathrm{D}$ and let $\mathscr{E}$ be the extension category of $\mathscr{C}$. By Corollary 4.4 D $\mathscr{E} \stackrel{\sim}{\longrightarrow} \mathbf{E}$ is a Waldhausen model for $\mathbf{E}$. Consider the following commutative diagram

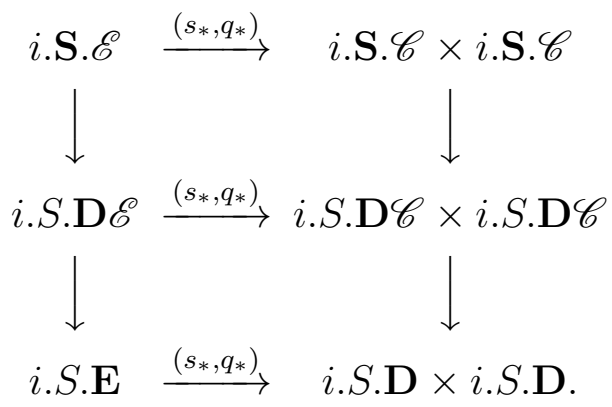

The vertical arrows are homotopy equivalences. By Corollary 3.9 the map $i . \mathbf{S} . \mathscr{E} \longrightarrow$ $i . \mathbf{S} . \mathscr{C} \times i . \mathbf{S} . \mathscr{C}$ is a homotopy equivalence. This implies the claim.

Let $\mathbf{D}$ be a dérivateur represented by a nice complicial biWaldhausen category. We can apply the $S$.-construction to each $\mathbf{S}_{n} \mathbf{D}$, obtaining a bisimplicial left pointed dérivateur represented by a nice complicial biWaldhausen category. Iterating this construction, we can form the multisimplicial object $\mathbf{S} .{ }^{n} \mathbf{D}=$ S.S. $\cdots$ S.D and the multisimplicial categories $i S .{ }^{n} \mathbf{D}$ of isomorphisms. The assertion below shows that $\left|i . S .{ }^{n} \mathbf{D}\right|$ is the loop space of $\left|i . S \cdot{ }^{n+1} \mathbf{D}\right|$ for any $n \geqslant 1$ and that the sequence

$$
\Omega|i . S . \mathbf{D}|, \Omega|i . S . S . \mathbf{D}|, \ldots, \Omega\left|i . S{ }^{n} \mathbf{D}\right|, \ldots
$$

forms a connective $\Omega$-spectrum KD (see the definition of the structure maps in [6]). In this case, one can think of the $K$-theory of $\mathbf{D}$ in terms of this spectrum. This does not affect the $K$-groups, because:

$$
\pi_{i}(\mathbf{K D})=\pi_{i}(K(\mathbf{D}))=K_{i}(\mathbf{D}), \quad i \geqslant 0
$$


Corollary 4.6. Let $\mathrm{D}$ be a dérivateur represented by a nice complicial biWaldhausen category. Then

$$
n \longmapsto i . S .{ }^{n} \mathbf{D}
$$

is a $\Omega$-spectrum beyond the first term. In particular, the $K$-theory of $\mathbf{D}$ can equivalently be defined as the space

$$
\Omega^{\infty}\left|i . S .{ }^{\infty} \mathbf{D}\right|=\lim _{n} \Omega^{n}\left|i . S .{ }^{n} \mathbf{D}\right| .
$$

Proof. For every $n \geqslant 0$, it follows from Lemma 4.2 that $\mathbf{S}_{n} \mathbf{D}$ is a dérivateur represented by a nice complicial biWaldhausen category. By Theorem 4.5 the class of such dérivateurs satisfies the addivity theorem. The claim now follows from [6, section 6].

Let us say a few words what thing goes wrong when conforming Waldhausen's [25] or McCarthy's [15] proof of additivity — in fact, both have the same complexity — to dérivateurs (or systems of diagram categories).

The first step is to show that additivity follows from the fact that for any Waldhausen category $\mathscr{C}$ the map

$$
s . \mathscr{E}(\mathscr{C}) \longrightarrow s . \mathscr{C} \times s . \mathscr{C}
$$

with $s . \mathscr{C}=\mathrm{Ob} S \mathscr{C}$ is a homotopy equivalence (just apply the same map to the Waldhausen category $w_{n} \mathscr{C}, n \geqslant 0$, of strings of weak equivalences and then apply Lemma 3.2). The same applies to left pointed dérivateurs: it suffices to show that for any left pointed dérivateur $\mathbf{D}$ the map

$$
s . \mathbf{E}(\mathbf{D}) \longrightarrow s . \mathbf{D} \times s . \mathbf{D}
$$

with $s . \mathbf{D}=\mathrm{Ob} S . \mathbf{D}$ is a homotopy equivalence. This is because the hyperfunctor $I \longmapsto$ $i_{n} \mathbf{D}_{I}$ taking an index category to the category of strings of isomorphisms is a left pointed dérivateur, too.

The second step consists of choices of pushouts (we neglect quotients here). Precisely, we are given two maps with common source $f: A \longrightarrow C$ and $\varphi: A \longrightarrow \bar{A}$ and $f$ a cofibration representing some simplex. Afterwards one constructs a pushout square

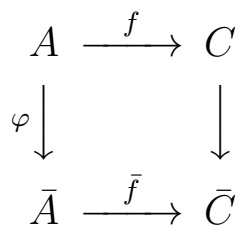

to get a simplex $\bar{A} \longmapsto \bar{C}$ from the simplex $A \longmapsto C$. However it is not immediately clear that the same procedure applies to dérivateurs. Normally we are given, as above, two objects $X, Y$ in $\mathbf{D}_{\Delta^{1}}$ with common "source" $X_{0}=Y_{0}$ and $X$ some simplex. To get a "new" simplex $\bar{X}$ from $X$ and $Y$ in the same way one should construct a cocartesian square whose projection on $(0,0) \longrightarrow(0,1)$ is the $X$ and that on $(1,0) \longrightarrow(1,1)$ is the $\bar{X}$. It seems that dérivateurs do not have enough information to do so. We were able to do that for dérivateurs represented by nice complicial biWaldhausen categories by using certain tricks of paragraph 2.3 , but it is not clear how to construct the necessary homotopy for all (left pointed) dérivateurs basing only on the known proofs of additivity for Waldhausen categories. 
To conclude this section, we would like to invite experts to prove additivity for dérivateurs represented by closed model categories. A similar technique used in this paper should be applicable for this case as well.

\section{THE DERIVED $K$-THEORY OF AN EXACT CATEGORY}

In this section we define the derived $K$-theory $D K(\mathscr{A})$ of an exact category $\mathscr{A}$. Though it is homotopy equivalent to the $K$-theory of its dérivateur $\mathbf{D}^{b}(\mathscr{A})$ it is more convenient for practical reasons to deal with the space $D K(\mathscr{A})$ than with the space $K\left(\mathbf{D}^{b}(\mathscr{A})\right)$.

Definition. The Algebraic DK-theory of an exact category $\mathscr{A}$ is defined as the pointed space

$$
D K(\mathscr{A})=\Omega|i \cdot \mathbf{S} \cdot \mathscr{A}| \text {. }
$$

The $D K$-groups of $\mathscr{A}$ are the homotopy groups of $D K(\mathscr{A})$

$$
D K_{*}(\mathscr{A})=\pi_{*}(\Omega|i . \mathbf{S} . \mathscr{A}|)=\pi_{*+1}(|i . \mathbf{S} \cdot \mathscr{A}|) .
$$

Let $(E x C a t s)$ denote the category of exact categories and exact functors. There results a functor

$$
D K:(\text { ExCats }) \longrightarrow(\text { Spaces })
$$

It follows from Corollary 4.3 that a natural map $D K(\mathscr{A}) \longrightarrow K\left(\mathbf{D}^{b}(\mathscr{A})\right)$ is a homotopy equivalence. Hence the $D K$-theories $D K(\mathscr{A})$ and $D K\left(\mathscr{A}^{\prime}\right)$ of exact categories $\mathscr{A}$ and $\mathscr{A}^{\prime}$ are homotopy equivalent whenever $K\left(\mathbf{D}^{b}(\mathscr{A})\right)$ and $K\left(\mathbf{D}^{b}\left(\mathscr{A}^{\prime}\right)\right)$ are. It also follows (see [6]) that $D K_{0}(\mathscr{A})$ is isomorphic to the Grothendieck group $K_{0}(\mathscr{A})$.

We prove below some basic results about $D K$-theory. The first result is the Additivity Theorem.

Theorem 5.1 (Additivity). Let $\mathscr{A}$ be an exact category and $\mathscr{E}$ its extension category. Then the map

$$
D K(s, q): D K(\mathscr{E}) \longrightarrow D K(\mathscr{A}) \times D K(\mathscr{A})
$$

is a homotopy equivalence. If $F^{\prime} \longrightarrow F \longrightarrow F^{\prime \prime}$ is an exact sequence of exact functors $\mathscr{A} \longrightarrow \mathscr{A}^{\prime}$ then there is a homotopy of maps

$$
D K(F) \simeq D K\left(F^{\prime}\right) \vee D K\left(F^{\prime \prime}\right): D K(\mathscr{A}) \longrightarrow D K\left(\mathscr{A}^{\prime}\right) .
$$

The DK-theory of $\mathscr{A}$ can equivalently be defined as the space

$$
\Omega^{\infty}\left|i . \mathbf{S} .^{\infty} \mathscr{A}\right|=\lim _{n} \Omega^{n}\left|i . \mathbf{S} .^{n} \mathscr{A}\right| .
$$

One can also think of the DK-theory in terms of the $\Omega$-spectrum

$$
\Omega \mid i . \text { S. } \mathscr{A}|, \Omega| i . \text { S.S. } \mathscr{A}|, \ldots, \Omega| i . \text { S. }^{n} \mathscr{A} \mid, \ldots
$$

Proof. These follow from results of section 3 
5.1. Approximation and Resolution theorems. In this paragraph we prove a modified Approximation Theorem and Resolution Theorem.

Theorem 5.2 (Approximation). Let $\mathscr{A}$ and $\mathscr{A}^{\prime}$ be two exact categories and let $w \mathscr{C}$ and $w \mathscr{C}^{\prime}$ denote the Waldhausen categories of quasi-isomorphisms in $\mathscr{C}=C^{b}(\mathscr{A})$ and in $\mathscr{C}^{\prime}=C^{b}\left(\mathscr{A}^{\prime}\right)$ respectively. Suppose further that $F: w \mathscr{C} \longrightarrow w \mathscr{C}^{\prime}$ is an exact functor of Waldhausen categories such that it induces an equivalence of the derived categories $D^{b}(\mathscr{A}) \stackrel{\sim}{\longrightarrow} D^{b}\left(\mathscr{A}^{\prime}\right)$. Then $D K(\mathscr{A})$ is homotopy equivalent to $D K\left(\mathscr{A}^{\prime}\right)$. If $F$ is induced by an exact functor $f: \mathscr{A} \longrightarrow \mathscr{A}^{\prime}$, this homotopy equivalence is given by the induced map $D K(f): D K(\mathscr{A}) \longrightarrow D K\left(\mathscr{A}^{\prime}\right)$.

We postpone the proof and first define some new concepts and prove certain technical lemmas.

Definition. Under the notation of Theorem 5.2 we say that $F$ has the approximation property (respectively h-approximation property) if it meets the axioms $A p p 1$ and $A p p 2$ below (respectively the axioms App1 and $H A p p 2$ ).

App 1 A map in $\mathscr{C}$ is a quasi-isomorphism if and only if its image is a quasi-isomorphism in $\mathscr{C}^{\prime}$.

App2 Any map $f: F X \longrightarrow Y$ in $\mathscr{C}^{\prime}, X \in \mathscr{C}$, fits into a commutative diagram

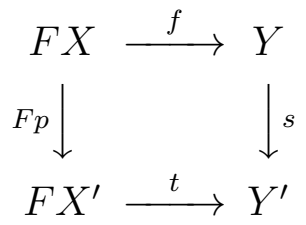

in which $p: X \longrightarrow X^{\prime}$ is a map in $\mathscr{C}$ and $s, t$ are quasi-isomorphisms in $\mathscr{C}^{\prime}$.

$H A p p 2$ Any map $f: F X \longrightarrow Y$ in $\mathscr{C}^{\prime}, X \in \mathscr{C}$, fits into a homotopy commutative diagram



with $s$ and $t$ quasi-isomorphisms in $\mathscr{C}^{\prime}$.

$F$ has the dual approximation property (respectively dual h-approximation property) if the axiom App1 and the dual axiom $A p p 2^{\text {op }}$ obtained by reversing the direction of arrows in $A p p 2$ (respectively the axiom $A p p 1$ and the dual axiom $H A p p 2^{\text {op }}$ ) are satisfied. These are a modification for the Waldhausen axioms $W A p p 1-W A p p 2$ [25, p. 352].

The next statement is due to Cisinski [1]. In fact, he proves it in a more general setting.

Proposition 5.3. Under the notation of Theorem 5.2 the following are equivalent:

(1) the functor $F$ induces an equivalence of the derived categories $D^{b}(\mathscr{A}) \longrightarrow D^{b}\left(\mathscr{A}^{\prime}\right)$;

(2) the functor $F$ has the approximation property;

(3) the functor $F$ has the h-approximation property;

$\left(2^{\mathrm{op}}\right)$ the functor $F$ has the dual approximation property;

$\left(3^{\mathrm{op}}\right)$ the functor $F$ has the dual h-approximation property. 
Proof. It is enough to show $(1) \Longleftrightarrow\left(2^{\text {op }}\right) \Longleftrightarrow\left(3^{\text {op }}\right)$ (the equivalences $(1) \Longleftrightarrow(2) \Longleftrightarrow$ (3) are dually proved).

$\left(2^{\mathrm{op}}\right) \Longrightarrow\left(3^{\mathrm{op}}\right)$ is obvious. Let us show $\left(3^{\mathrm{op}}\right) \Longrightarrow\left(2^{\mathrm{op}}\right)$. Any arrow $f: Y \longrightarrow F X$ can be fitted into the following diagram:

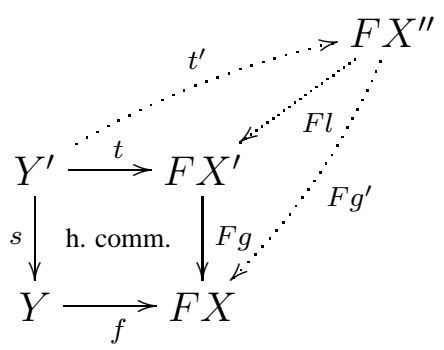

where the homotopy commutative square with the entries $\left(Y^{\prime}, F X^{\prime}, Y, F X\right)$ exists by assumption, $X^{\prime \prime}=\operatorname{Cocyl}(g)$ and the square with the entries $\left(Y^{\prime}, F X^{\prime \prime}, Y, F X\right)$ is commutative (see paragraph 2.3).

$(1) \Longrightarrow\left(3^{\mathrm{op}}\right)$. The axiom $A p p 1$ is obvious. Check the axiom $H A p p 2$. Let $\alpha: Y \longrightarrow$ $F X$ be an arrow in $\mathscr{C}^{\prime}$. There exists an isomorphism $t s^{-1}: F X^{\prime} \longrightarrow Y$ in $D^{b}\left(\mathscr{A}^{\prime}\right)$ resulting a map $F X^{\prime} \longrightarrow F X$. Let this map be the image of a map $X^{\prime} \longrightarrow X$ in $D^{b}(\mathscr{A})$ represented by a diagram $X^{\prime} \stackrel{q}{\longleftarrow} X^{\prime \prime} \stackrel{f}{\longrightarrow} X$.

There is a common denominator

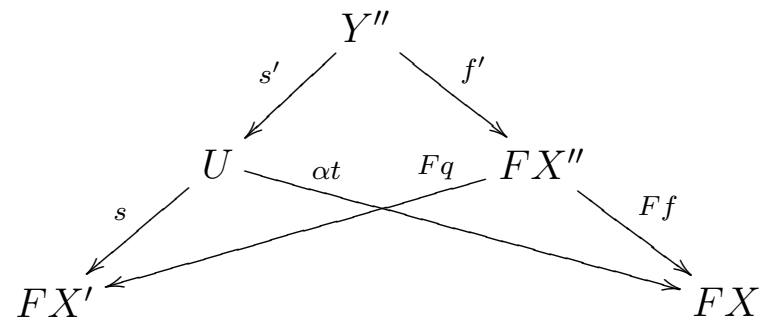

yielding a homotopy commutative square

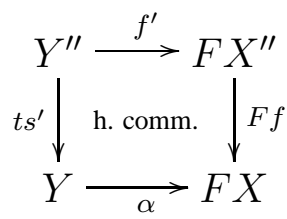

It remains thus to show $\left(2^{\mathrm{op}}\right) \Longrightarrow(1)$. Given an object $Y \in D^{b}\left(\mathscr{A}^{\prime}\right)$ there is a diagram

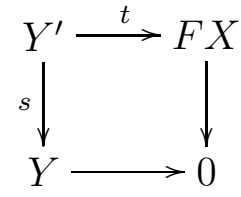

with $s, t$ quasi-isomorphisms. We see that $D^{b}(\mathscr{A}) \longrightarrow D^{b}\left(\mathscr{A}^{\prime}\right)$ is essentially surjective. Let us show that it is full. 
Let $\alpha: F X \longrightarrow F X^{\prime}$ be a map in $D^{b}\left(\mathscr{A}^{\prime}\right)$ represented by a diagram $F X \stackrel{s}{\longleftarrow} Y \stackrel{f}{\longrightarrow}$ $F X^{\prime}$. There is a commutative diagram



with $q, t$ quasi-isomorphisms. It follows that $F u$ is a quasi-isomorphism. We get a denominator

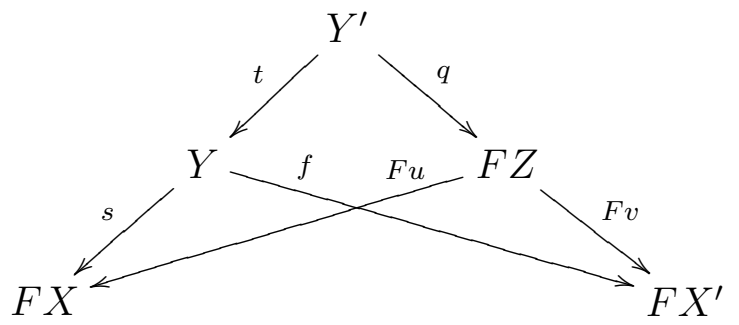

This shows that $\alpha$ is the image of $v u^{-1}: X \longrightarrow X^{\prime}$.

To show that the functor in question is faithful we shall need the following

Sublemma. Let two maps $u, v: X \longrightarrow Y$ in $\mathscr{C}$ be such that there is a quasi-isomorphism $q: U \longrightarrow F X$ and $F u \circ q$ and $F v \circ q$ are homotopic in $\mathscr{C}^{\prime}$. Then there exists a quasiisomorphism $s: T^{\prime} \longrightarrow X$ in $\mathscr{C}$ such that us is homotopic to vs.

Proof. Let $\operatorname{Cocyl}(Y)$ denote the cocylinder of the map $1_{Y}$ and write $d_{0}, d_{1}: \operatorname{Cocyl}(Y) \longrightarrow$ $Y$ for the natural projections. The map $\left(d_{0}, d_{1}\right): \operatorname{Cocyl}(Y) \longrightarrow Y \times Y$ is an epimorphism in $\mathscr{C}$. Construct a cartesian diagram

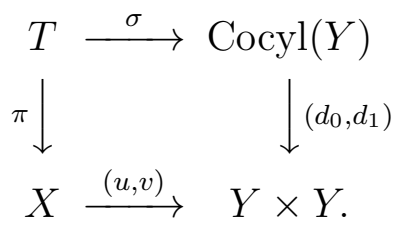

Since $F u \circ q, F v \circ q$ are homotopic, there is a map $\alpha: U \longrightarrow F[\operatorname{Cocyl}(Y)]=\operatorname{Cocyl}(F Y)$ such that $\left(F d_{0}, F d_{1}\right) \circ \alpha=(F u, F v) \circ q$. There results a commutative diagram

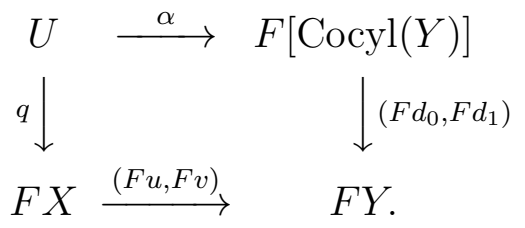

There is a unique map $v: U \longrightarrow F T$ such that $F(\sigma) \circ v=\alpha$ and $F(\pi) \circ v=q$.

The map $v$ fits into a commutative square

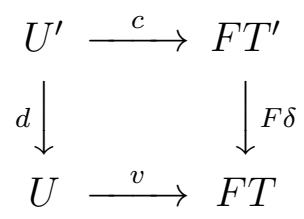


with $c, d$ quasi-isomorphisms. We see that $F(\pi \delta) \circ c=q d$ is a quasi-isomorphism, and so is $F(\pi \delta)$. By $A p p 1$ the map $\pi \delta$ is a quasi-isomorphism. It follows that $u(\pi \delta)$ is homotopic to $v(\pi \delta)$, hence the required map $s$ is $\pi \delta$.

Now prove that the functor $D^{b}(\mathscr{A}) \longrightarrow D^{b}\left(\mathscr{A}^{\prime}\right)$ is faithful. It suffices to show that if $F f=0$ in $D^{b}\left(\mathscr{A}^{\prime}\right)$ with $f: X \longrightarrow Y$ a map in $\mathscr{C}$, then $f$ equals to zero in $D^{b}(\mathscr{A})$.

The property for a map $\alpha$ in $D^{b}\left(\mathscr{A}^{\prime}\right)$ of being equal to zero is equivalent to saying that there is a quasi-isomorphism $q$ such that $\alpha q$ is homotopic to zero. By our assumption $F f \circ q \sim 0$, and so there exists a quasi-isomorphism $s$ in $\mathscr{C}$ with $f s \sim 0$ by the sublemma above. It follows that $f$ equals to zero in $D^{b}(\mathscr{A})$. We are done.

The last proposition also applies to nice complicial biWaldhausen categories $\mathscr{A}$ and $\mathscr{B}$ and a complicial exact functor between them. The following shows to which extent the Thomason Approximation Theorem [22, 1.9.8] for nice complicial biWaldhausen categories is a modification of the Waldhausen Approximation Theorem [25, 1.6.7]

Corollary 5.4. Let $\mathscr{A}$ and $\mathscr{B}$ be nice complicial biWaldhausen categories and let $F$ : $\mathscr{A} \longrightarrow \mathscr{B}$ be a complicial exact functor. Suppose that $F$ has the approximation or the h-approximation property (the axioms App 1 - App 2 or App 1 - H App2). Then F induces a homotopy equivalence of $K$-theory spaces

$$
K(F): K(\mathscr{A}) \longrightarrow K(\mathscr{B}) .
$$

Proof. By Proposition $5.3 F$ induces an equivalence of the derived categories $w^{-1} F$ : $w^{-1} \mathscr{A} \longrightarrow w^{-1} \mathscr{B}$. Then $K(F)$ is a homotopy equivalence by [22, 1.9.8].

Lemma 5.5. An exact functor $F: w \mathscr{C} \longrightarrow w \mathscr{C}^{\prime}$ meets the axiom App2 if and only if any arrow $f: F X \longrightarrow Y$ in $\mathscr{C}^{\prime}, X \in \mathscr{C}$, fits into a commutative diagram

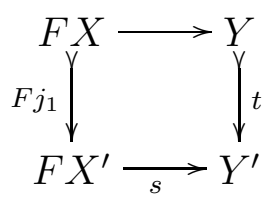

in which $j_{1}, t$ are cofibrations and $s, t$ are quasi-isomorphisms in $\mathscr{C}^{\prime}$.

Proof. If $F$ meets the axiom $A p p 2$, then any arrow $f: F X \longrightarrow Y$ in $\mathscr{C}^{\prime}$ fits into a commutative diagram

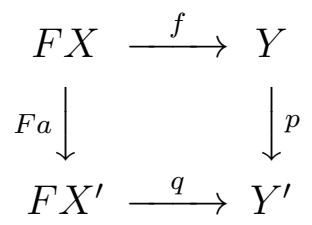

in which $p, q$ are quasi-isomorphisms. Let $T=\operatorname{Cyl}(a)$, then $a=r j_{1}$ with $j_{1}: X \longrightarrow T$ a cofibration and $r: T \longrightarrow X^{\prime}$ a quasi-isomorphism. Construct a cocartesian square

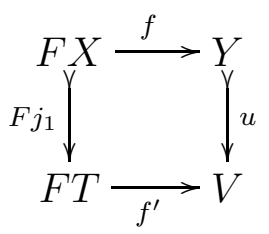


There exists a unique map $v: V \longrightarrow Y^{\prime}$ such that $q \circ F r=v f^{\prime}$ and $v u=p$. The map $v$ factors as $V \stackrel{m}{\longrightarrow} T^{\prime} \stackrel{n}{\longrightarrow} Y^{\prime}$ with $T^{\prime}=\mathrm{Cyl}(v), m$ a cofibration and $n$ a quasiisomorphism. There results a commutative diagram

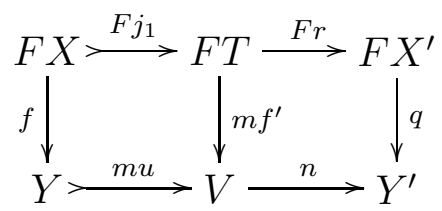

Since $p=n m u$ and $p, n$ are quasi-isomorphisms, we see that $m u$ is a quasi-isomorphism. Also, $m f^{\prime}$ is a quasi-isomorphism because $n, q, F r$ are. We are done.

Proof of Theorem 5.2 In view of Lemma 3.2 and Proposition 5.2 it suffices to check that for any $n \geqslant 0$ the induced functor $w S_{n} \mathscr{C} \longrightarrow w S_{n} \mathscr{C}^{\prime}$ has the approximation property. This is so for $n=0$. Obviously, it is enough to check the approximation property for the map $w F_{n} \mathscr{C} \longrightarrow w F_{n} \mathscr{C}^{\prime}$ and $n \geqslant 0$. For $n=0$ it follows from our assumption and Proposition 5.2 If we show this for $n=1$ the general case will follow by induction.

Let the diagram represent a map $a: F X \longrightarrow Y$ in $F_{1} \mathscr{C}=C^{b}\left(F_{1} \mathscr{A}\right)$,

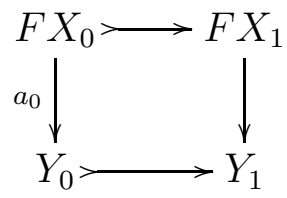

Then $a_{0}$ fits into a commutative square

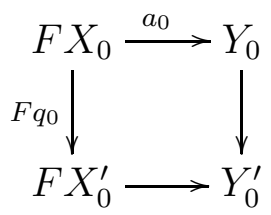

One can construct the following commutative diagram

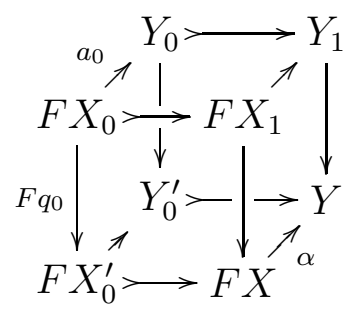

with $X=X_{0}^{\prime} \coprod_{X_{0}} X_{1}$ and $Y=Y_{0}^{\prime} \coprod_{Y_{0}} Y_{1}$. By Lemma 5.5 $\alpha$ fits into a commutative diagram

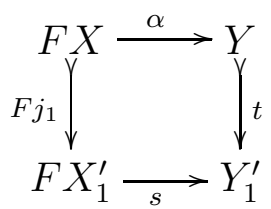


in which $j_{1}, t$ are cofibrations and $s, t$ are quasi-isomorphisms in $\mathscr{C}^{\prime}$. We get the commutative diagram

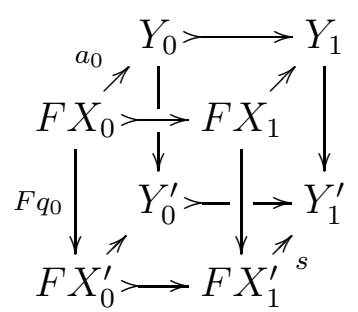

that shows $A p p 2$. The axiom $A p p 1$ is obvious. The theorem is proved.

Theorem 5.6 (Resolution). Let $\mathscr{P}$ be an extension closed full exact subcategory of an exact category $\mathscr{M}$. Assume further that

(1) if $M^{\prime} \longmapsto M \rightarrow M^{\prime \prime}$ is exact in $\mathscr{M}$ and $M, M^{\prime \prime} \in \mathscr{P}$, then $M^{\prime} \in \mathscr{P}$ and

(2) for any object $M \in \mathscr{M}$ there is a finite resolution $0 \longrightarrow P_{n} \longrightarrow P_{n-1} \longrightarrow \cdots \longrightarrow$ $P_{0} \longrightarrow M \longrightarrow 0$ with $P_{i} \in \mathscr{P}$.

Then $D K(\mathscr{P}) \longrightarrow D K(\mathscr{M})$ is a homotopy equivalence (and thus $D K_{i}(\mathscr{P}) \simeq D K_{i}(\mathscr{M})$ for all $i$ ).

Proof. By [11, 12.1] an extension closed full exact subcategory $\mathscr{P}$ of an exact category $\mathscr{M}$ induces a fully faithful canonical functor between their bounded derived categories if for any exact sequence $M^{\prime \prime} \longmapsto M^{\prime} \rightarrow P$ in $\mathscr{M}$ with $P \in \mathscr{P}$, there is a commutative diagram

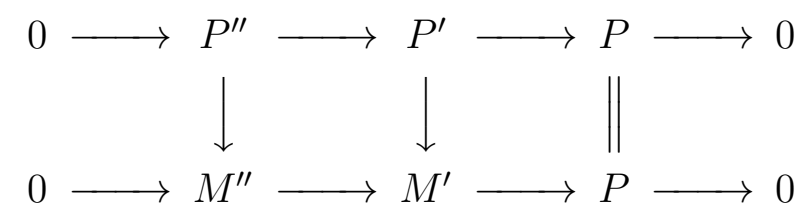

with $P^{\prime}, P^{\prime \prime} \in \mathscr{P}$ and in which the first row is also exact. This condition follows from our assumptions. Indeed, by (2) one can choose an admissible epimorphism $P^{\prime} \longrightarrow M^{\prime}$ and the kernel of the composed map $P^{\prime} \longrightarrow P$ is in $\mathscr{P}$ by (1).

Since each object $M \in \mathscr{M}$ has a finite resolution by objects in $\mathscr{P}$, it follows that for every bounded complex $A$ with entries in $\mathscr{M}$ there exists a quasi-isomorphism $B \longrightarrow A$ for some bounded complex $B$ with entries in $\mathscr{P}$ (the proof is dual to that of [10, 4.1(b)]). Therefore, the canonical functor $D^{b}(\mathscr{P}) \longrightarrow D^{b}(\mathscr{M})$ is an equivalence. Theorem 5.2 now implies the claim.

5.2. Pairings. Let $\mathscr{A}, \mathscr{B}, \mathscr{C}$ be exact categories. We want to pair Quillen's $K$-theory of $\mathscr{A}$ and the derived $K$-theory of $\mathscr{B}$ into the derived $K$-theory of the latter. The appropriate assumption to make is a pairing

$$
f: \mathscr{A} \times \mathscr{B} \longrightarrow \mathscr{C}
$$

which is a biexact functor, that is for each $A \in \mathscr{A}$ and $B \in \mathscr{B}$ the partial functors

$$
f(A,-): \mathscr{B} \longrightarrow \mathscr{C}, \quad f(-, B): \mathscr{A} \longrightarrow \mathscr{C}
$$

are exact. We shall think of $f$ as a tensor product. For technical reasons we assume that each of $\mathscr{A}, \mathscr{B}, \mathscr{C}$ is equipped with a distinguished zero object 0 and that $f(A, 0)=0=$ $f(0, B)$ always. Such a $f$ gives rise a pairing

$$
f: \mathscr{A} \times C^{b}(\mathscr{B}) \longrightarrow C^{b}(\mathscr{C})
$$


which is also a biexact functor.

Let $s . \mathscr{A}$ denote the simplicial set $\left\{\mathrm{Ob} S_{n} \mathscr{A}\right\}_{n}$. We obtain a map

$$
|s . \mathscr{A}| \times \mid i . \text { S. } \mathscr{B}|\longrightarrow| i \text {.S.S. } \mathscr{C} \mid
$$

that takes $|s . \mathscr{A}| \vee|i . \mathbf{S} . \mathscr{B}|$ into the basepoint of $\mid i$.S.S. $\mathscr{C} \mid$ because of the technical assumption we made. This yields a map

$$
|s . \mathscr{A}| \wedge|i . \mathbf{S} . \mathscr{B}| \longrightarrow \mid i . \text { S.S. } \mathscr{C} \mid
$$

and hence a map

$$
\Omega|s . \mathscr{A}| \wedge \Omega \mid i . \text { S. } \mathscr{B}|\longrightarrow \Omega \Omega| i . \text { S.S. } \mathscr{C} \mid \text {. }
$$

This is the desired pairing in $K$-theory in view of the homotopy equivalence of $\Omega|s . \mathscr{A}|$ with $K(\mathscr{A})$ and $\Omega \Omega \mid i$.S.S. $\mathscr{C} \mid$ with $D K(\mathscr{C})$. So we get a map of spaces

$$
K(\mathscr{A}) \wedge D K(\mathscr{B}) \longrightarrow D K(\mathscr{C})
$$

and hence homomorphisms of abelian groups

$$
K_{m}(\mathscr{A}) \otimes D K_{n}(\mathscr{B}) \longrightarrow D K_{m+n}(\mathscr{C}), \quad m, n \geqslant 0 .
$$

5.3. Conjectures. The central problem here is comparison of $D K(\mathscr{A})$ with Quillen's $K$-theory $K(\mathscr{A})$. There is a natural map $K(\rho): K(\mathscr{A}) \longrightarrow D K(\mathscr{A})$ factoring as

$$
K(\mathscr{A}) \stackrel{K(\tau)}{\longrightarrow} K\left(w C^{b}(\mathscr{A})\right) \stackrel{K(\nu)}{\longrightarrow} D K(\mathscr{A})
$$

where $w C^{b}(\mathscr{A})$ stands for the category of quasi-isomorphisms in $C^{b}(\mathscr{A})$ and $K\left(w C^{b}(\mathscr{A})\right)$ is its Waldhausen $K$-theory. The map on the left is induced by the map taking an object $A \in \mathscr{A}$ to the complex concentrated in the zeroth degree and the map $\nu$ is induced by the quotient functor $C^{b}(\mathscr{A}) \longrightarrow D^{b}(\mathscr{A})$.

Question (The first Maltsiniotis conjecture [14]). The map $K(\rho): K(\mathscr{A}) \longrightarrow D K(\mathscr{A})$ is a homotopy equivalence.

Let $\mathscr{A}$ admit an exact fully faithful functor $i: \mathscr{A} \longrightarrow \mathscr{B}$ with $\mathscr{B}$ an abelian category such that for any map $f$ in $\mathscr{A}$ with $i(f)$ an epimorphism in $\mathscr{B}$ the map $f$ is an epimorphism. This is the case when weak idempotent object split in $\mathscr{A}$ (see [22]). Then $K(\tau)$ is a homotopy equivalence by the Gillet-Waldhausen theorem [22, 1.11.7]. In this case, the comparison conjecture is reduced to showing that $K(\nu)$ is a homotopy equivalence.

Let us consider the composed map of spaces

$$
K(f): K(\mathscr{A}) \stackrel{K(\rho)}{\longrightarrow} D K(\mathscr{A}) \stackrel{K(\mu)}{\longrightarrow} K\left(\mathbf{D}^{b}(\mathscr{A})\right)
$$

in which the right arrow is a homotopy equivalence. It is shown in [6] that $K_{0}(f)$ is an isomorphism and hence is so $K_{0}(\rho): K_{0}(\mathscr{A}) \longrightarrow D K_{0}(\mathscr{A})$.

I personally doubt that the comparison conjecture is true. This is caused by a recent observation of Toën and Vezzosi [23]: the obvious generalization of this conjecture to all Waldhausen categories can not be true for obvious functoriality reasons. It is true for the Waldhausen $K$-theory of spaces, for example.

Now we want to formulate a sort of Localization Theorem for the $D K$-theory. We think that the following ingredients would be the most reasonable to do that. 
(1) One should first find the relevant notions of a thick exact subcategory $\mathscr{A}$ of an exact category $\mathscr{U}$ and a quotient exact category $\mathscr{U} / \mathscr{A}$ satisfying the obvious universal property in $(E x C a t s)$.

(2) If $\mathscr{A} \subseteq \mathscr{U}$ is thick then so is $S_{n} \mathscr{A} \subseteq S_{n} \mathscr{U}$ for every $n$.

(3) If $\mathscr{A}$ is thick and idempotent complete in $\mathscr{U}$ then the sequence of bounded derived categories

$$
D^{b}(\mathscr{A}) \longrightarrow D^{b}(\mathscr{U}) \longrightarrow D^{b}(\mathscr{U} / \mathscr{A})
$$

is an exact sequence of triangulated categories, i.e. $D^{b}(\mathscr{A})$ is the full triangulated category of $D^{b}(\mathscr{U})$ on objects zero in $D^{b}(\mathscr{U} / \mathscr{A})$ and $D^{b}(\mathscr{U}) / D^{b}(\mathscr{A})=D^{b}(\mathscr{U} / \mathscr{A})$.

The desired notions are suggested by Schlichting in [19] (see also his Dissertationsschrift [17]). The conditions (1)-(2) are satisfied for $\mathscr{A} \subseteq \mathscr{U}$ whenever $\mathscr{A}$ is a "left or right s-filtering subcategory" in $\mathscr{U}$ in the sense of [19] (for brevity thick) and if, moreover, $\mathscr{A}$ is idempotent complete, then (3) is also valid. For example, any filtering subcategory in the sense of Karoubi [9] or Pedersen-Weibel [16] is thick. Notice that if all categories considered are abelian, then any thick subcategory is Serre.

Question (Localization). If $\mathscr{A}$ is a thick and idempotent complete subcategory of an exact category $\mathscr{U}$ then the sequence of exact categories $\mathscr{A} \longrightarrow \mathscr{U} \longrightarrow \mathscr{U} / \mathscr{A}$ induces a homotopy fibration of spaces

$$
D K(\mathscr{A}) \longrightarrow D K(\mathscr{U}) \longrightarrow D K(\mathscr{U} / \mathscr{A}) .
$$

Localization would follow if we showed that the quotient functor $\mathscr{U} \longrightarrow \mathscr{U} / \mathscr{A}$ induces a homotopy equivalence

$$
i . S . S .(\mathscr{A} \subset \mathscr{U}) \longrightarrow i . S . S .(0 \subset \mathscr{U} / \mathscr{A}) .
$$

Indeed, we would have then the following commutative diagram

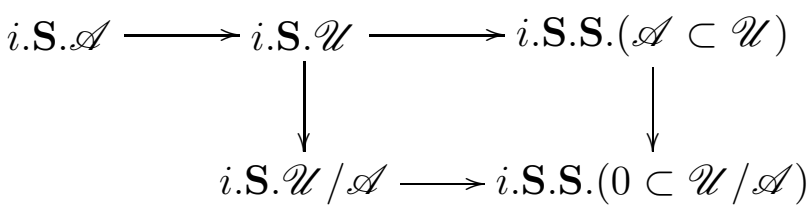

in which the first horizontal line is a homotopy fibration by Corollary 3.8. The right arrow is a homotopy equivalence and $i$.S. $\mathscr{U} / \mathscr{A} \longrightarrow i$. S.S. $(0 \subset \mathscr{U} / \mathscr{A})$ is a homotopy equivalence, too (for example by appealing again to Corollary 3.8 ).

\section{ADDENDUM}

In this section we give the definition of a left pointed dérivateur and its $K$-theory. The theory of dérivateurs was developed by Grothendieck in [7]. Very close to dérivateurs objects (the so-called "homotopy theories" and "systems of diagrams categories") have been studied by Heller [8] and Franke [5]. Since this paper mostly deals with the dérivateur given by the hyperfunctor

$$
I \longmapsto \mathbf{D}^{b}\left(\mathscr{A}^{I}\right)
$$

where $\mathscr{A}$ is an exact category we will only discuss dérivateurs and its $K$-theory space although the analogous $K$-theory can also be defined for systems of diagram categories (see [6] for details). 
6.1. The axioms. For the notions of the 2-category and 2-functor we refer the reader to [13]. In what follows we use the term "poset" as an abbreviation of "finite partially ordered set". The 2-category of the posets (respectively the finite categories without cycles) we shall denote by $\mathcal{O}$ rd (respectively by $\mathcal{D}$ irf).

Let $\mathcal{D} i a$ be a full 2-subcategory of the 2-category Cat of small categories that contains the 2-category $\mathcal{O}$ rd. We assume that Dia satisfies the following conditions:

(1) Dia is closed under finite sums and finite products;

(2) for any functor $f: I \longrightarrow J$ in Dia and for any object $y$ of $J$, the categories $f / y$ and $f \backslash y$ are in $\mathcal{D} i a$.

We shall also refer to $\mathcal{D} i$ a a category of diagrams.

A predérivateur of the domain $\mathcal{D} i a$ or just a predérivateur is a functor

$$
\mathrm{D}: \mathcal{D} i a^{\mathrm{op}} \longrightarrow \mathrm{CAT}
$$

from Dia to the category CAT of categories (not necessarily small) satisfying the Functoriality Axiom below. So to each category $I$ in $\mathcal{D} i a$ there is associated a category $\mathbf{D}_{I}$, and to each map $f: I \longrightarrow J$ in Dia a functor $f^{*}=\mathbf{D}(f): \mathbf{D}_{J} \longrightarrow \mathbf{D}_{I}$.

Functoriality Axiom. The following conditions hold:

$\diamond$ to each natural transformation $\varphi: f \longrightarrow g$ a natural transformation $\varphi^{*}: f^{*} \longrightarrow$ $g^{*}$ is associated and the maps $f \longrightarrow f^{*}$ and $\varphi \longrightarrow \varphi^{*}$ define a functor from $\operatorname{Hom}(I, J)$ to the category of functors from $\mathbf{D}_{J}$ to $\mathbf{D}_{I}$;

$\diamond$ if

$$
K \stackrel{f}{\longrightarrow} I \underset{g^{\prime}}{\stackrel{g}{\longrightarrow}} J \stackrel{h}{\longrightarrow} L
$$

are morphisms and $\varphi: g \longrightarrow g^{\prime}$ is a bimorphism, then $f^{*} \circ \varphi^{*}=(\varphi \circ f)^{*}$ and $\varphi^{*} \circ h^{*}=(h \circ \varphi)^{*}$.

A morphism $F: \mathbf{D} \longrightarrow \mathbf{D}^{\prime}$ between two predérivateurs $\mathbf{D}$ and $\mathbf{D}^{\prime}$ consists of the following data:

(1) for any $I \in \mathcal{D} i a$, a functor $F: \mathbf{D}_{I} \longrightarrow \mathbf{D}_{I}^{\prime}$;

(2) for any map $f: I \longrightarrow J$ in $\mathcal{D} i a, f^{*} F=F f^{*}$;

(3) for any bimorphism $\varphi: f \longrightarrow g$ in $\mathcal{D} i a, \varphi^{*} F=F \varphi^{*}$.

A morphism $F: \mathbf{D} \longrightarrow \mathbf{D}^{\prime}$ is an equivalence if for any $I \in \mathcal{D} i a$ the functor $F$ : $\mathbf{D}_{I} \longrightarrow \mathbf{D}_{I}^{\prime}$ is an equivalence of categories.

Given $I \in \mathcal{D} i a$ and $x \in I$, let $i_{x, I}: 0 \longrightarrow I$ be the functor sending 0 to $x$. For $A \in \mathbf{D}_{I}$ let $A_{x}=i_{x, I}^{*} A$. Let us consider the following axioms listed below.

Isomorphism Axiom. A morphism $f: A \longrightarrow B$ in $\mathbf{D}_{I}$ is an isomorphism if and only if $f_{x}: A_{x} \longrightarrow B_{x}$ is so for all $x \in I$.

Disjoint Union Axiom. (a) If $I=I_{1} \coprod I_{2}$ is a disjoint union of its full subcategories $I_{1}$ and $I_{2}$, then the inclusions $i_{1 ; 2}: I_{1 ; 2} \longrightarrow I$ define an equivalence of categories

$$
\left(i_{1}^{*}, i_{2}^{*}\right): \mathbf{D}_{I} \stackrel{\sim}{\longrightarrow} \mathbf{D}_{I_{1}} \times \mathbf{D}_{I_{2}} .
$$

(b) $\mathbf{D}_{\emptyset}$ is a trivial category (having one morphism between any pair of objects). 
Homotopy Kan Extension Axiom. The left homotopy Kan extension axiom says that for any functor $f: I \longrightarrow J$, the functor $f^{*}: \mathbf{D}_{J} \longrightarrow \mathbf{D}_{I}$ has a left adjoint $f_{!}: \mathbf{D}_{I} \longrightarrow \mathbf{D}_{J}$.

Base Change Axiom. Let $f: I \longrightarrow J$ be a morphism in Dia and $x \in J$. Consider the diagram in Dia

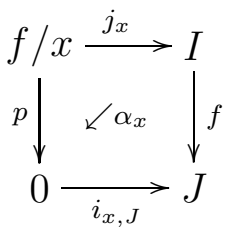

with $j_{x}$ a natural map and $\alpha_{x}$ the natural bimorphism. The $\alpha_{x}$ induces a bimorphism $\beta_{x}: p_{!} j_{x}^{*} \longrightarrow i_{x, I}^{*} f_{!}$. The left base change axiom requires $\beta_{x}$ to be an isomorphism.

Definition. A predérivateur is called a left dérivateur if the Functoriality Axiom, the Isomorphism Axiom, the Disjoint Union Axiom, the Left Homotopy Kan Extension Axiom, and the Left Base Change Axiom are satisfied.

Let $F: \mathbf{D} \longrightarrow \mathbf{D}^{\prime}$ be a morphism between two left dérivateurs, and let $f: I \longrightarrow J$ be a map in Dia. Consider the adjunction maps $\alpha: 1 \longrightarrow f^{*} f_{!}$and $\beta: f_{!} f^{*} \longrightarrow 1$. Denote by $\gamma_{F, f}$ the composed map

$$
f_{!} F \stackrel{f_{!} F \alpha}{\longrightarrow} f_{!} F f^{*} f_{!}=f_{!} f^{*} F f_{!} \stackrel{\beta F f_{!}}{\longrightarrow} F f_{!} .
$$

$F$ is right exact if $\gamma_{F, f}$ is an isomorphism and the following compatibility relations hold:

$$
F \alpha_{\mathbf{D}}=f^{*}\left(\gamma_{F, f}\right) \circ \alpha_{\mathbf{D}^{\prime}} F \text { and } F \beta_{\mathbf{D}}=\beta_{\mathbf{D}^{\prime}} F \circ \gamma_{F, f}^{-1} f^{*} .
$$

The dérivateurs we work with are also to meet some extra conditions. A map $f: I \longrightarrow$ $J$ in Dia is a closed (open) immersion if it is a fully faithful inclusion such that for any $x \in J$ the relation $\operatorname{Hom}(I, x) \neq \emptyset(\operatorname{Hom}(x, I) \neq \emptyset)$ implies $x \in I$.

Definition. A left dérivateur is pointed if the following conditions hold:

(1) for any closed immersion $f: I \longrightarrow J$ in Dia, the structure functor $f$ ! possesses a left adjoint $f^{\text {? }}$;

(2) for any open immersion $f: I \longrightarrow J$ in $\mathcal{D} i a$, the structure functor $f^{*}$ possesses a right adjoint $f_{*}$;

(3) for any open immersion $f: I \longrightarrow J$ in Dia and any object $x \in J$, the base change morphism of the diagram

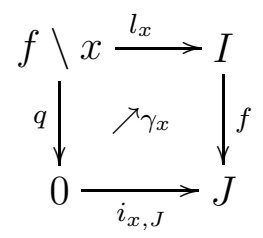

yields an isomorphism $\delta_{x}: i_{x, I}^{*} f_{*} \longrightarrow q_{*} l_{x}^{*}$.

If $\mathbf{D}$ is a left pointed dérivateur, then $\mathbf{D}_{I}$ has a zero object for any $I \in \mathcal{D} i a$. 
6.2. The $S$-construction and $K$-theory space. Throughout this section $\mathbf{D}$ is assumed to be a left pointed dérivateur (of the domain $\mathcal{D} i a$ ).

Let $\square \in \mathcal{D}$ ia be the poset $\Delta^{1} \times \Delta^{1}$ and let $\Gamma \subset \square$ be the subposet $\square \backslash(1,1)$. Let $i_{\ulcorner}:\left\ulcorner\longrightarrow \square\right.$ be the inclusion. An object $A$ of $\mathbf{D}_{\square}$ is called cocartesian if the canonical morphism $i_{\ulcorner,} i_{\ulcorner}^{*} A \longrightarrow A$ is an isomorphism.

Given $0 \leqslant i<j<k \leqslant n$ let

$$
a_{i, j, k}: \square \longrightarrow \operatorname{Ar} \Delta^{n}
$$

denote the functor defined as:

$$
(0,0) \longmapsto(i, j), \quad(0,1) \longmapsto(i, k), \quad(1,0) \longmapsto(j, j), \quad(1,1) \longmapsto(j, k) .
$$

For any integer $n \geqslant 0$, denote by $S_{n} \mathbf{D}$ the full subcategory of $\mathbf{D}_{\mathrm{Ar} \Delta^{n}}$ that consists of the objects $X$ satisfying the following two conditions:

$\diamond$ for any $i \leqslant n, X_{(i, i)}$ is isomorphic to zero in $\mathbf{D}_{0}$;

$\diamond$ for any $0 \leqslant i<j<k \leqslant n, a_{i, j, k}^{*} X$ is a cocartesian square if $n>1$.

The definition of $S_{n} \mathbf{D}$ is similar to that of $S_{n} \mathscr{C}$, where $\mathscr{C}$ is a Waldhausen category [25]. $S_{0} \mathbf{D}$ is the full subcategory of zero objects in $\mathbf{D}_{0}$. The category $S_{1} \mathbf{D}$ consists of the objects $X \in \mathbf{D}_{\Delta^{2}}$ with $X_{0}$ and $X_{2}$ isomorphic to zero.

For any object $I$ of $\mathcal{D} i a$, we denote by $\mathbf{D}(I)$ the left pointed dérivateur defined as $\mathbf{D}(I)_{J}=\mathbf{D}_{I \times J}$. Let $\mathbf{S}_{n} \mathbf{D}_{I}=S_{n} \mathbf{D}(I)$. Then $\mathbf{S}_{n} \mathbf{D}$ is a left pointed dérivateur (see [6]). There results a simplicial left pointed dérivateur

$$
\text { S.D : } \Delta^{n} \longmapsto \mathbf{S}_{n} \text { D. }
$$

Consider the following simplicial category:

$$
\text { S.D : } \Delta^{n} \longmapsto S_{n} \mathbf{D} \text {. }
$$

For any $n \geqslant 0$, let $i S_{n} \mathbf{D}$ denote the subcategory of $S_{n} \mathbf{D}$ whose objects are those of $S_{n} \mathbf{D}$ and whose morphisms are isomorphisms in $S_{n} \mathbf{D}$, and let $i . S_{n} \mathbf{D}$ be the nerve of $i S_{n} \mathbf{D}$. We obtain then the following bisimplicial object:

$$
\text { i.S. : } \Delta^{m} \times \Delta^{n} \longmapsto i_{m} S_{n} \mathbf{D} .
$$

Definition. The Algebraic K-theory for a small left pointed dérivateur $\mathbf{D}$ of the domain $\mathcal{D} i a$ is given by the pointed space (a fixed zero object 0 of $\mathbf{D}_{0}$ is taken as a basepoint)

$$
K(\mathbf{D})=\Omega|i . S . \mathbf{D}|
$$

The $K$-groups of $\mathbf{D}$ are the homotopy groups of $K(\mathbf{D})$

$$
K_{*}(\mathbf{D})=\pi_{*}(\Omega|i . S . \mathbf{D}|)=\pi_{*+1}(|i . S . \mathbf{D}|) .
$$

Denote by (Left pointed dérivateurs) the category of left pointed dérivateurs and right exact functors. Then the map

$$
\text { (Left pointed dérivateurs) } \stackrel{K}{\longrightarrow} \text { (Spaces) }
$$

is functorial.

Let $\mathbf{D}$ be a left pointed dérivateur. Denote by $\mathbf{E}_{0}$ the full subcategory in $\mathbf{D}_{\square}$ consisting of the cocartesian squares $E \in \mathbf{D}_{\square}$ with $E_{(1,0)}$ isomorphic to zero. If we replace $\mathbf{D}$ by $\mathbf{D}(I)$, we define the category $\mathbf{E}_{I}$ similar to $\mathbf{E}_{0}$. One obtains a left pointed dérivateur $\mathbf{E}$. It is equivalent (by a right exact map) to the dérivateur $\mathbf{S}_{2} \mathbf{D}[6,6.2]$. 


\section{REFERENCES}

[1] D.-C. Cisinski, Catégories dérivables, preprint, 2002.

[2] D.-C. Cisinski, An e-interchange, January 2004.

[3] W. G. Dwyer, D. M. Kan, Simplicial localizations of categories, J. Pure Appl. Algebra 17(3) (1980), 267-284.

[4] W. G. Dwyer, D. M. Kan, Calculating simplicial localizations, J. Pure Appl. Algebra 18(1) (1980), 17-35.

[5] J. Franke, Uniqueness theorems for certain triangulated categories with an Adams spectral sequence, K-theory Preprint Archives 139 (1996).

[6] G. Garkusha, Systems of diagram categories and K-theory. I, preprint, 2003.

[7] A. Grothendieck, Les Dérivateurs, manuscript, 1990.

[8] A. Heller, Homotopy theories, Mem. Amer. Math. Soc. 71 (1988), No. 383.

[9] M. Karoubi, Foncteurs dérivés et K-théorie, In Séminaire Heidelberg-Saarbrücken-Strasbourg sur la K-théorie 1967/68, Lecture Notes in Mathematics, No. 136, Springer-Verlag, 1970, pp. 107-186.

[10] B. Keller, Chain complexes and stable categories, Manus. Math. 67 (1990), 379-417.

[11] B. Keller, Derived categories and their uses, In Handbook of Algebra, vol. 1, North-Holland, Amsterdam, 1996, pp. 671-701.

[12] B. Keller, Le dérivateur triangulé associé à une catégorie exacte, preprint, 2002.

[13] S. Mac Lane, Categories for the working mathematician, Graduate Texts in Mathematics 5, SpringerVerlag, New-York, 1998. Second edition.

[14] G. Maltsiniotis, La K-théorie d'un dérivateur triangulé, preprint, 2002.

[15] R. McCarthy, On fundamental theorems of algebraic K-theory, Topology 32(2) (1993), 325-328.

[16] E. K. Pedersen, C. A. Weibel, K-theory homology of spaces, In Algebraic topology, Arcata/CA 1986, Lecture Notes in Mathematics, No. 1370, Springer-Verlag, 1989, pp. 346-361.

[17] M. Schlichting, Délaçage de la K-théorie des catégories exactes et K-groupes négatifs, Thése, Université Paris 7, 2000.

[18] M. Schlichting, A note on K-theory and triangulated categories, Inv. Math. 150 (2002), 111-116.

[19] M. Schlichting, Delooping the K-theory of exact categories, Topology, to appear.

[20] G. Segal, Categories and cohomology theories, Topology 13 (1974), 293-312.

[21] R. E. Staffeldt, On fundamental theorems of algebraic K-theory, K-theory 1 (1989), 511-532.

[22] R. W. Thomason, T. Trobaugh, Higher algebraic K-theory of schemes and of derived categories, The Grothendieck Festschrift III, Progress in Mathematics 88, Birkhäuser, 1990, pp. 247-435.

[23] B. Toën, G. Vezzosi, Remark on K-theory and S-categories, Topology 43(4) (2004), 765-791.

[24] F. Waldhausen, Algebraic K-theory of generalized free products, Ann. Math. 108 (1978), 135-256.

[25] F. Waldhausen, Algebraic K-theory of spaces, In Algebraic and geometric topology, Proc. Conf., New Brunswick/USA 1983, Lecture Notes in Mathematics, No. 1126, Springer-Verlag, 1985, pp. 318-419.

International Centre for Theoretical Physics, Strada Costiera 11, I-34014, Trieste, ITALY

URL: www.ictp.trieste.it/ garkusha

E-mail address: ggarkusha@mail.ru 